\title{
Unfolding the multiscale structure of networks with dynamical Ollivier-Ricci curvature
}

Adam Gosztolai ( $\nabla$ adam.gosztolai@epfl.ch )

École Polytechnique Fédérale de Lausanne https://orcid.org/0000-0002-0699-5825

Alexis Arnaudon

Imperial College London

Article

Keywords: network structure, learning algorithms, network processes, Ollivier-Ricci curvature

Posted Date: February 16th, 2021

DOl: https://doi.org/10.21203/rs.3.rs-222407/v1

License: (9) This work is licensed under a Creative Commons Attribution 4.0 International License. Read Full License

Version of Record: A version of this preprint was published at Nature Communications on July 27th, 2021. See the published version at https://doi.org/10.1038/s41467-021-24884-1. 


\footnotetext{
*corresponding author: adam.gosztolai@epfl.ch

${ }^{\dagger}$ Current address: Blue Brain Project, École polytechnique fédérale de Lausanne (EPFL), Campus Biotech, 1202 Geneva, Switzerland
}

\section{Unfolding the multiscale structure of networks with dynamical Ollivier-Ricci curvature}

\author{
Adam Gosztolai $^{* 1}$ and Alexis Arnaudon ${ }^{\dagger 2}$ \\ ${ }^{1}$ Neuroengineering Laboratory, Brain Mind Institute $\mathscr{6}$ Interfaculty Institute of Bioengineering, \\ EPFL, Lausanne, Switzerland \\ ${ }^{2}$ Department of Mathematics, Imperial College London, London, United Kingdom
}

\begin{abstract}
Defining the geometry of networks is typically associated with embedding in low-dimensional spaces such as manifolds. This approach has helped design efficient learning algorithms, unveil network symmetries and study dynamical network processes. However, the choice of embedding space is network-specific, and incompatible spaces can result in information loss. Here, we define a dynamic edge curvature for the study of arbitrary networks measuring the similarity between pairs of dynamical network processes seeded at nearby nodes. We show that the evolution of the curvature distribution exhibits gaps at characteristic timescales indicating bottleneck-edges that limit information spreading. Importantly, curvature gaps robustly encode communities until the phase transition of detectability, where spectral clustering methods fail. We use this insight to derive geometric modularity optimisation and demonstrate it on the European power grid and the $C$. elegans homeobox gene regulatory network finding previously unidentified communities on multiple scales. Our work suggests using network geometry for studying and controlling the structure of and information spreading on networks.
\end{abstract}

Real-world networks are rarely embedded in physical or Euclidean spaces, which complicates their analysis. However, to correctly represent node similarities, it is typical to assume that the network's nodes lie in a low-dimensional subspace, such as a manifold or linear subspace ${ }^{1}$. Having this geometric backbone permits the efficient functioning of standard clustering methods, including ones based on Euclidean geometric features such as k-means or expectation maximisation ${ }^{2}$. A related means of geometrising networks is possible by embedding nodes into a continuous space. For example, the hyperbolic space of constant negative curvature provides a natural parametrisation of complex networks to unveil their self-similar clusters across scales ${ }^{3,4}$. Likewise, embedding networks into a geometric space based on a suitable distance metric between dynamical network processes has helped reveal their functional organisation ${ }^{5,6}$. However, in general, there is no guarantee that a network is compatible with a given metric space without suffering significant distortion ${ }^{7}$. At the same time, a network may have several, not necessarily self-similar, geometric representations arising, for example, from clusters at multiple resolutions ${ }^{8}$. Thus, there is a need for a geometric notion that does not require embedding, yet allows studying the multiscale structure of a general class of networks.

A promising candidate is the Ollivier-Ricci (OR) curvature $^{9}$, which measures the change of local connectivity from one node to another, given by the cost of transporting a unit of mass between their respective neighbourhoods. Instead of imposing a geometry on the network through embedding, the OR curvature induces an effective geometry that has precise interpretation in limiting cases. In fact, it is the only one among a number of discrete curvature notions ${ }^{10,11}$ known to converge rigorously to the traditional Ricci curvature of a Riemannian manifold ${ }^{12}$. The OR curvature is also related to graph theoretical objects, including the local clustering coefficient and bounds on the spectrum of the graph Laplacian ${ }^{13,14}$, and has 
lead to advances in applications such as studying the robustness of economic networks ${ }^{15}$, characterising the human brain structural connectivity ${ }^{16}$ and designing clustering heuristics ${ }^{17,18}$.

However, several properties of the OR curvature hinder its widespread applicability to study network clusters. Since it depends on structural neighbourhoods, it lacks a resolution parameter to define a geometry on different resolutions, that is necessary to study the multiscale structure in real-world networks. Further, the OR curvature of an edge is a local quantity, in a sense that it is controlled by the degree of its endpoints ${ }^{13}$. Thus, it may provide a suboptimal geometric representation of sparse networks - including many real-world networks where each node connects only to a few others - in which node degrees vary widely. This lack of robustness of the OR curvature for sparse networks also precludes its use for studying from a geometric perspective the phase transition occurring as the community structure gets weaker and become abruptly undetectable ${ }^{19-21}$. In other words, there is a need for a geometric notion that does not rely on embeddings, robustly captures multiscale clusters in real networks, and captures the phase transition at the limit of cluster detection.

\section{Results}

\section{Dynamical Ollivier-Ricci curvature from graph diffusion}

We address this need by combining two distinct frameworks - network-driven dynamical processes and geometry with OR curvature. The spreading of network-driven dynamical processes is shaped by the heterogeneity of the network. In turn, one may infer the network structure by observing properties of their evolution. We focus on Markov diffusion processes ${ }^{8,22-24}$, a class of linear dynamical systems which is rich enough to capture several properties of nonlinear processes on networks ${ }^{25,26}$. On a connected network $G$ weighted by pairwise distances $w_{i j}$, the continuous time diffusion is constructed by the standard procedure ${ }^{27}$ of defining the normalised graph Laplacian matrix $\mathbf{L}:=\mathbf{K}^{-1}(\mathbf{K}-\mathbf{A})$, where $\mathbf{K}$ is the diagonal matrix of node degrees with $K_{i i}=\sum_{j} A_{i j}$ and $\mathbf{A}$ is the weighted adjacency matrix encoding similarities between nodes. For example, one may simply take $A_{i j}=\max _{i j} w_{i j}-w_{i j}$, or $A_{i j}=e^{-w_{i j}}$. Then, the probability measure of the diffusion started from the unit mass $\delta_{i}$ on node $i$ (Fig. 1a, b) evolves according to

$$
\mathbf{p}_{i}(\tau)=\delta_{i} e^{-\tau \mathbf{L}} .
$$

In analogy to the Ricci curvature on a manifold, the classical OR curvature ${ }^{9,28}$ measures the change of one-step neighbourhoods between nodes (see Methods and Supplementary Fig. 1a for background). Here, instead of structural neighbourhoods we consider distributions generated by diffusion processes across scales $\tau$. Specifically, we start a diffusion process at each node $i=1, \ldots, n$ to obtain a set of measures $\mathbf{p}_{i}(\tau)$. We then define the dynamic Ollivier-Ricci curvature of an edge as the distance of adjacent pairs of measures relative to the distance of their starting points

$$
\kappa_{i j}(\tau):=1-\frac{\mathcal{W}_{1}\left(\mathbf{p}_{i}(\tau), \mathbf{p}_{j}(\tau)\right)}{w_{i j}},
$$

whenever $i j$ is an edge and 0 otherwise. Intuitively, Eq. (2) measures how much 'closer' diffusions get over time when started $w_{i j}$ distance apart, measured by $\mathcal{W}_{1}$, the optimal transport distance ${ }^{29}$. It is obtained as a solution to a minimisation problem (Eq. (13) in Methods) and encodes the least cost of transporting the measure $\mathbf{p}_{i}(\tau)$ to $\mathbf{p}_{j}(\tau)$ via the edges on the graph. The minimiser of this problem is the optimal transport plan represented as a matrix $\boldsymbol{\zeta}(\tau)$. The entries of this matrix shown on Fig. 1c, d quantify how much mass is moved between each pair of nodes $u$ and $v$ along their connecting geodesic of length $d_{u v}$ (Fig. 1e).

As expected, our definition recovers the classical OR curvature ${ }^{9}$ as a first-order approximation for small times $\tau \ll 1$. Indeed, $\mathbf{p}_{i}(\tau) \simeq \delta_{i} \mathbf{K}^{-1} \mathbf{A}$ is the one-step measure encoding the local connectivity. Further, the dynamical OR curvature inherits the geometric intuition of the classical definition. Notably, on canonical trees-like and cliques-like networks the $\kappa_{i j}(\tau)$ is negative and positive, respectively, for all finite scales $\tau$ analogously to the Ricci curvature on hyperboloids and spheres (Supplementary Fig. 1b, c). 

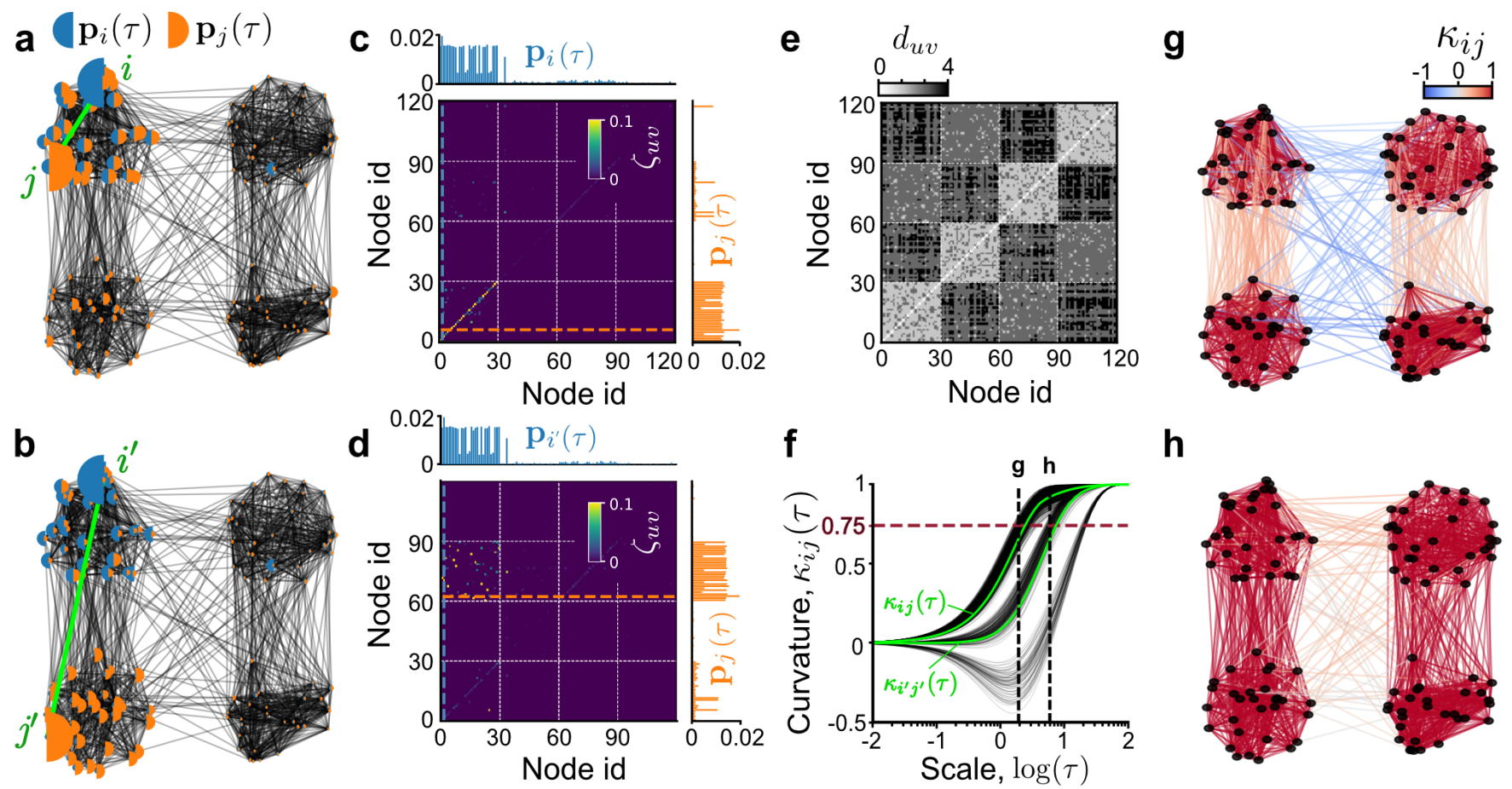

Figure 1: Dynamical Ollivier-Ricci curvature capturing the spreading of diffusion processes. a Snapshot at time $\log \tau=0.15$ of a pair of diffusion measures $\mathbf{p}_{i}(\tau)$ and $\mathbf{p}_{j}(\tau)$ started at nodes $i, j$ of a stochastic block model network ( $n=120$ with four equal clusters with edge probabilities 0.8 within clusters and 0.1 or 0.02 between clusters). When $i$ and $j$ are in the same cluster, the measures overlap significantly. The size of half-circles is proportional to the amount of mass on the respective nodes. $\mathbf{b}$ For $i^{\prime}, j^{\prime}$ in different clusters the measures remain largely disjoint. c Optimal transport plan $\zeta(\tau)$ superimposed with $\mathbf{p}_{i}(\tau), \mathbf{p}_{j}(\tau)$. When $i, j$ (colored dashed lines) lie in the same cluster only diagonal elements $\zeta_{u u}$ are positive, meaning only geodesics within a cluster transport significant mass. The white dashed lines correspond to the four clusters. d Same as $\mathbf{c}$, but with diffusions started at nodes $i^{\prime}$ and $j^{\prime}$ in different clusters. Only entries $\zeta_{u v}$ with $u$ and $v$ in different clusters have significant nonzero weight. e Geodesic distance matrix showing the block structure of the network. $\mathbf{f}$ The evolution of the edge curvatures (Eq. (2)) against time, with the highlighted lines corresponding to edges in $\mathbf{a}, \mathbf{b}$. Here $\kappa_{i j}(\tau) \simeq 0.75$ indicates scales when local mixing occurs between diffusion pairs. The dashed vertical lines show two such scales $(\log \tau=0.15,0.43)$. $\mathbf{g}, \mathbf{h}$ Graph edges coloured by the curvature reveals the clusters at the two scales.

In the following we are interested in studying the curvature distribution across edges when the network structure deviates from these canonical topologies.

\section{Edge curvature gap differences in rate of information spreading}

Most real-world networks exhibit organisation on several scales. As an illustration, the unweighted stochastic block model (SBM) network ${ }^{30}$ of four equal-size clusters contains two nontrivial scales if the edges are drawn independently with probability 0.8 within clusters and 0.1 or 0.02 between clusters (Fig. 1a, b). We show that multiscale structure can be revealed by scanning through a finite range of scales $\tau$ and studying snapshots of curvature distribution across edges.

The characteristic scales of a network are related to the overlap between pairs of diffusion measures $\mathbf{p}_{i}(\tau), \mathbf{p}_{j}(\tau)$. This overlap depends on the starting points $i, j$ and on network clusters which can confine diffusions on well-connected regions for long times before reaching the stationary state $\boldsymbol{\pi}^{8,22,23,31}$, with $\pi_{i}=\mathbf{K}_{i i} / \sum_{i} \mathbf{K}_{i i}$. This transient phenomenon is reflected by the structure of the optimal transport matrix $\boldsymbol{\zeta}(\tau)$. If $i, j$ lie within the same subnetwork, the measures quickly overlap (Fig. 1a) and only diagonal entries of $\boldsymbol{\zeta}(\tau)$ are positive (Fig. 1c), weighing only short, within-cluster geodesics. By contrast, started at different subnetworks, the measures remain almost disjoint (Fig. 1b) and $\boldsymbol{\zeta}(\tau)$ is forced to select longer 
geodesics (Fig. 1d, e), reflected by the large entries in the off-diagonal block.

The evolution of the edge curvature $\kappa_{i j}(\tau)$ (Fig. 1f) aggregates the information in $\boldsymbol{\zeta}(\tau)$ into a single number that is related to the rate of mass exchange between subnetworks at a given scale. We see in Fig. If that, initially, when all nodes support disjoint point masses and the diffusions have not yet mixed, $\lim _{\tau \rightarrow 0} \kappa_{i j}(\tau) \rightarrow 1-\mathcal{W}_{1}\left(\delta_{i}, \delta_{j}\right) / d_{i j}=0$. At the other extreme, as the diffusions reach stationary state, $\lim _{\tau \rightarrow \infty} \kappa_{i j}(\tau) \rightarrow 1-\mathcal{W}_{1}(\boldsymbol{\pi}, \boldsymbol{\pi}) / d_{i j}=1$. At intermediate scales, the curvature can take values between 1 and some finite negative number depending on the graph. We find that, as the curvature of an edge evolves, the scale at which it approaches unity indicates how easy it is to propagate information between the subnetworks. More precisely, in the Methods, we prove that this scale gives an upper bound on the mixing time $\tau_{i j}^{\text {mix }}$ of the diffusion pair, namely,

$$
\begin{aligned}
\tau_{i j}^{\text {mix }} & :=\frac{1}{2} \sum_{u v}\left|\zeta_{u v}(\tau)-\zeta_{u v}(\infty)\right| \\
& \leq \min \left\{\tau: \kappa_{i j}(\tau) \geq 0.75\right\}
\end{aligned}
$$

where $\boldsymbol{\zeta}(\tau)$ is the optimal transport plan with marginals $\mathbf{p}_{i}(\tau)$ and $\mathbf{p}_{j}(\tau)$. Note that $\kappa_{i j}(\tau) \geq 0.75$ does not imply that the corresponding diffusion processes have approached stationary state independently, but only that they exchange negligible mass at that or larger scales.

Importantly, a gap in the distribution of curvatures appears when the curvature exceeds 0.75 for some edges while remaining less than 0.75 for others indicating a network bottleneck that limits mass flow. To illustrate this, Fig. 1f shows three groups of edges, those with most positive curvature are found within clusters, while the other two groups of edges are found between pairs of clusters. Figs. 1g, h correspond to two scales on Fig. If $(\log \tau=0.15,0.43)$ where the curvature has exceeded 0.75 for some groups of edges, indicating the diffusions are well mixed within these groups, but not across other edges for which the curvature is less than 0.75 . The latter mark bottleneck edges which lie between the expected partitions with 4 and 2 clusters, respectively. This simple example shows the importance of the scale parameter $\tau$ in our curvature definition to capture the network structure at multiple scales. Before applying this to real networks, we take a closer look at the curvature gap in the theoretical context of the stochastic block model.

\section{Curvature gap is a robust indicator of clusters in stochastic block models}

Since in our example any pair of diffusions are supported by one (Fig. 1a) or two (Fig. 1b) clusters, we focus on studying the subgraph $G$ induced by two clusters (Fig. 2a). The subgraph $G$ is a realisation of $\mathcal{G}=\operatorname{SSBM}\left(n / 2, p_{\text {in }}, p_{\text {out }}\right)$, the symmetric SBM composed of two planted partitions of equal size. Edges are generated independently with probability $p_{\text {in }}$ within-clusters and probability $p_{\text {out }}$ between-clusters. We will denote the ground truth with $C_{i} \in\{1,-1\}$ for each node $i$ and define $\bar{k}=n\left(p_{\text {in }}+p_{\text {out }}\right) / 2$ as the average degree.

Classical spectral clustering methods ${ }^{27}$ perform well for dense graphs (Fig. 2a), where $\bar{k}$ is an increasing function of $n$. This suppresses fluctuations for large $n$ causing a spectral gap to appear when the eigenvalue $\lambda_{c}$ of the Laplacian matrix $\mathbf{L}$ of $G$ separates from bulk eigenvalues arising from randomness ${ }^{27}$ (Fig. 2c). In this dense regime, $\lambda_{c}$ is well approximated by $\left\langle\lambda_{c}\right\rangle_{\mathcal{G}}=2 p_{\text {out }} /\left(p_{\text {in }}+p_{\text {out }}\right)$, the second eigenvalue of the ensemble averaged Laplacian $\langle\mathbf{L}\rangle_{\mathcal{G}}$ (see Supplementary Note 1). Since $\lambda_{c}$ can be identified due to the spectral gap, clustering involves simply labelling nodes by the sign of the entries of the corresponding eigenvector, which also approximates the ensemble average eigenvector $\phi_{c}(u)=1 / \sqrt{n}$ when $C_{u}=1$ and $-1 / \sqrt{n}$ when $C_{u}=-1$. However, for sparse graphs (Fig. 2b), where $\bar{k}$ is constant (independent of $n$ ), the spectral gap ceases to exist ${ }^{32}$ (Fig. 2d). Thus, spectral algorithms relying on identifying $\lambda_{c}$ perform no better than chance. To perform clustering in this regime, one needs to go beyond spectral clustering using, for example, the belief propagation method in statistical physics or the related non-backtracking operator whose spectrum is better behaved ${ }^{19,21}$. 
a
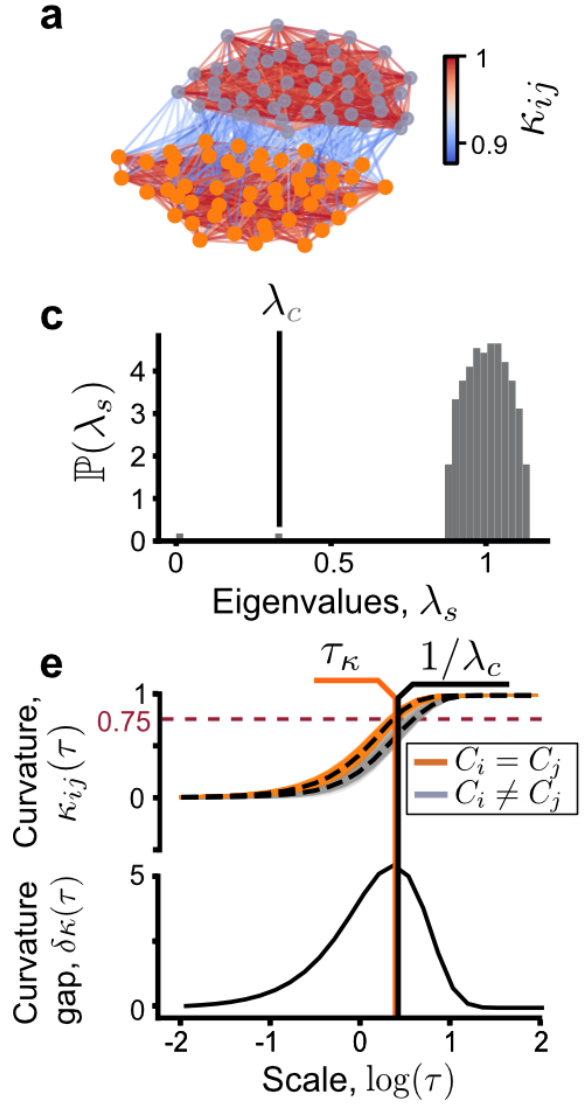

b
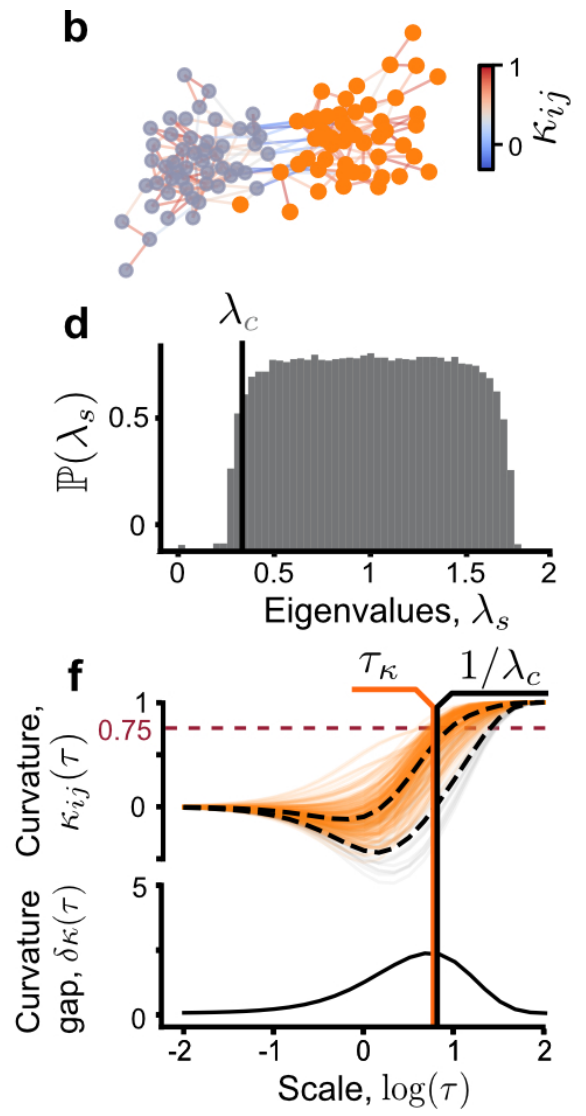

g

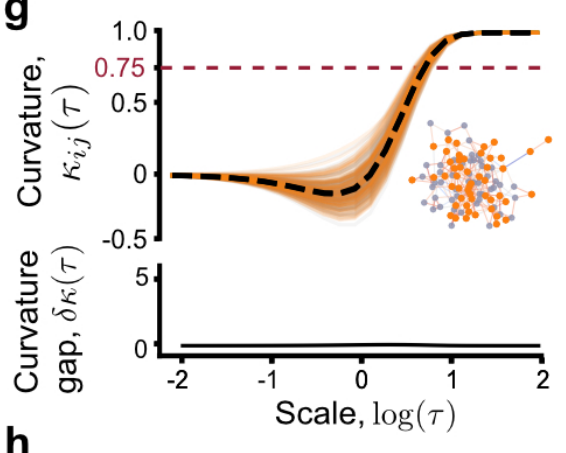

h

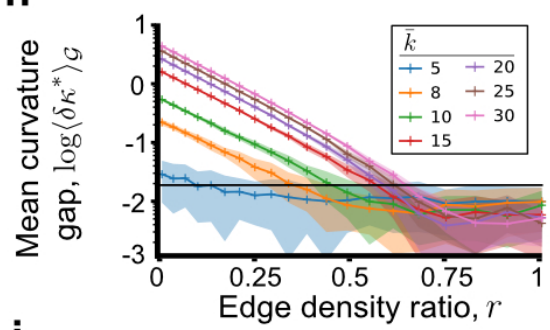

i

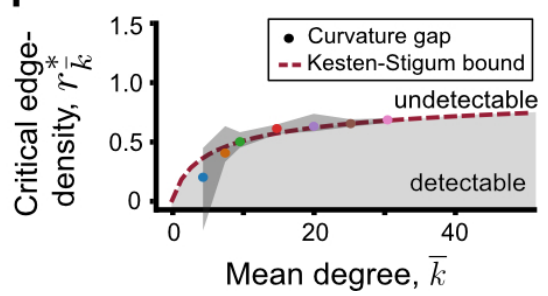

Figure 2: Edge curvature gap indicates the presence of clusters where spectral clustering fails. Twopartition symmetric SBM graph in the a dense regime $\left(p_{\text {in }}=0.5, p_{\text {out }}=0.1\right)$ and $\mathbf{b}$ sparse regime $\left(p_{\text {in }}=8 / n, p_{\text {out }}=\right.$ $0.5 / n)$. Edges are coloured by the curvature $(n=100, \log \tau=0.83)$. $\mathbf{c}, \mathbf{d}$ The histogram of eigenvalues obtained from five SBM realisations in dense and sparse regime, respectively. In the dense regime, the eigenvalue $\lambda_{c}$ corresponding to the community structure is well separated from the bulk eigenvalues, but overlaps in the sparse regime. e, f The evolution of edge curvatures driven against diffusion time. A gap between the curvatures of within-edges and between-edges is associated with the presence of clusters. When $\kappa_{i j}>0.75$ (horizontal dashed line) the diffusions are well mixed across the respective edges. The curvature gap is maximal at $\tau_{\kappa} \approx \lambda_{c}^{-1}$ (orange and black vertical lines). $\mathrm{g}$ There is no curvature gap in the limiting ER graph (inset, $\left.p_{\text {in }}=p_{\text {out }}=(8+0.5) /(2 n)\right)$. $\mathbf{h}$ Maximal curvature gap averaged over $20 \mathrm{SBM}$ realisations for each fixed $\bar{k}$ with $10^{4}$ nodes, against edge density ratio. The horizontal line marks the estimated background noise level. The intersection of this line with the mean curvature gap defines $r_{\bar{k}}^{*}$, the largest possible edge density ratio to detect clusters. i Phase diagram of critical edge density ratio against average degree. The numerically obtained critical edge density ratios computed from the curvature gap are superimposed with the theoretical Kesten-Stigum detection limit (dashed line) and show excellent agreement. Gray shaded area denotes the regime where detection is possible.

To see how robustly the dynamical OR curvature indicates the presence of clusters in the symmetric SBM, let us construct a measure on the curvature evolution. To this end, we define the curvature gap as the difference between the mean curvatures of within- and between-edges

$$
\delta \kappa(\tau):=\frac{1}{\sigma}\left|\left\langle\kappa_{i j}(\tau)\right\rangle_{C_{i}=C_{j}}-\left\langle\kappa_{i j}(\tau)\right\rangle_{C_{i} \neq C_{j}}\right|
$$

where the averages are on within and between-edges, normalised by $\sigma=\sqrt{\frac{1}{2}\left(\sigma_{\text {within }}^{2}+\sigma_{\text {between }}^{2}\right)}$ in terms of the standard deviations of both sets of curvatures. This measure is adapted from the sensitivity index in signal detection theory, known to be, asymptotically, the most powerful statistical test for discriminating two distributions ${ }^{33}$. Large curvature gap $\delta \kappa(\tau)$ indicates that the within and between edges have curvatures different enough for the clusters to be recovered (Fig. 2e, f). Correspondingly, in the limits $\tau \rightarrow 0, \infty$ 

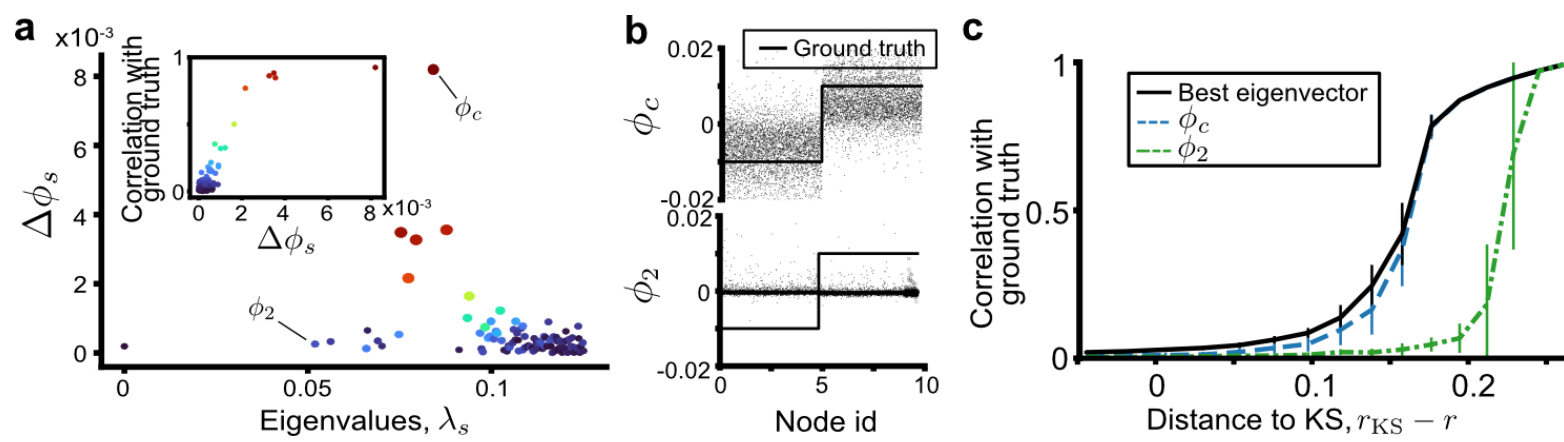

Figure 3: Detecting communities using pairs of diffusions near the weak recovery limit. a Difference in eigenvectors $\Delta \phi_{s}$ (Eq. (8)) between diffusion processes started at adjacent nodes for a single sparse SBM network $\left(p_{\text {in }}=3 / n, p_{\text {out }}=0.5 / n, n=10^{4}\right)$. Each dot marks $\left(\lambda_{s}, \Delta \phi_{s}\right)$ for the 50 smallest eigenvectors, colored by the correlation of the corresponding eigenvector with the ground truth, shown in the inset. $\mathbf{b}$ The eigenvector with the highest $\Delta \phi_{s}$ encodes the cluster structure (solid line), whereas the second eigenvector $\phi_{2}$, used by spectral clustering methods, are driven by high random fluctuations. c Correlation of eigenvectors with ground truth against distance to KS limit $\left(n=10^{5}, \bar{k}=3\right)$. The eigenvector identified by the highest $\Delta \phi_{s}$ approaches the correlation with the ground truth of the best eigenvector in the spectrum. All eigenvectors become uncorrelated with the ground truth at the KS limit.

where the curvatures are uniform across the graph $\delta \kappa(\tau)$ vanishes and, likewise, in the absence of structure ( $p_{\text {in }} \approx p_{\text {out }}$ in the Erdös-Rényi (ER) limit) we have $\delta \kappa(\tau)=0$ for all $\tau$ (Fig. $2 \mathrm{~g}$ ). At intermediate scales, we find that the scale of maximal curvature gap occurs at $\tau_{\kappa}$ at which point the curvatures of within-edges is $\kappa_{i j}\left(\tau_{\kappa}\right) \approx 0.75$. In agreement with Eq. (3), this indicates well-mixed diffusions across these edges relative to low-curvature bottleneck edges between clusters, which indicate incomplete mixing. We also find that $\tau_{\kappa} \approx \lambda_{c}^{-1}$ (Fig. 2e, f). These results show that positive curvature gap is associated with the presence of clusters.

What is the minimum curvature gap needed to detect clusters? Previous works on the limits of cluster detection has shown that if the clusters are too weak (high $r:=p_{\text {out }} / p_{\text {in }}$ ) or the graph too sparse (low $\bar{k}$ ), no algorithm can assign the vertices to communities better than chance, or distinguish $G$ from an Erdős-Rényi graph $(r=1)$. This is known as the limit of weak-recovery or detection and is characterised by the Kesten-Stigum (KS) threshold $r=r_{\mathrm{KS}}=(\bar{k}-\sqrt{\bar{k}}) /(\bar{k}+\sqrt{\bar{k}})^{19,20,34}$.

To study this limit, we sampled 20 networks from $\mathcal{G}$ for a range of $\bar{k}$ and $r$. For each sample, we computed the maximal curvature gap $\delta \kappa^{*}:=\max _{\tau} \delta \kappa(\tau)$ and formed the ensemble average quantity $\left\langle\delta \kappa^{*}\right\rangle_{\mathcal{G}}$. As $r$ increases for a given $\bar{k}$ we observe that $\left\langle\delta \kappa^{*}\right\rangle_{\mathcal{G}}$ decreases exponentially until a certain noise level (Fig. $2 \mathrm{~h})$. The critical edge density ratio $r_{\bar{k}}^{*}$ can be estimated as the smallest $r$ where $\left\langle\delta \kappa^{*}\right\rangle_{\mathcal{G}}$ dropped below a threshold background noise level, estimated here at 0.035 (black horizontal line). This choice of threshold is not absolute, as it is affected by the finite-size effect of the SBM graphs. An analytical derivation of this threshold is out of scope of this work, but our numerical experiment clearly shows that the curvature gap detects a signal from the planted partitions up to the KS limit (Fig. 2i).

\section{Geometric cluster detection in the sparse regime}

Given that the curvature gap (Eq. (4)) indicates the presence of clusters until the fundamental KS limit we asked if this information could be used to recover the ground truth partition. The definition of curvature gap (Eq. (4)) suggests looking for equilibrium configurations of the unit-temperature Boltzmann distribution over the cluster assignments $C$,

$$
\mathbb{P}(C \mid \boldsymbol{\kappa}) \propto e^{\sum_{i j} \kappa_{i j}(\tau) \delta\left(C_{i}, C_{j}\right)}
$$

where $\boldsymbol{\kappa}$ is a matrix with entries $\kappa_{i j}$ and the sum is over all edges $i j$. The distribution involves only within-edges because finding those is equivalent to finding between-edges, up to a normalisation factor. 
The distribution $\mathbb{P}(C \mid \boldsymbol{\kappa})$ is important because all of its equilibrium states are equivalent and correlate with the ground truth partition of the symmetric SBM $\mathcal{G}$. To see this, we connect $\mathbb{P}(C \mid \boldsymbol{\kappa})$ to the posterior distribution $\mathbb{P}(C \mid G)$ of the cluster assignments obtained given the graph drawn from $\mathcal{G}$. In the sparse regime, the likelihood of observing $G$ with a given cluster assignment $C$ is

$$
\mathbb{P}(G \mid C) \propto \prod_{i j}\left(\frac{p_{\text {in }}}{p_{\text {out }}}\right)^{\delta\left(C_{i}, C_{j}\right)} \propto \mathbb{P}(C \mid G)
$$

(see Eq. (17) in Methods). The second part of Eq. (6) results from Bayes' theorem using a uniform prior on $C$, since a priori all configurations are equally likely. It has been previously shown ${ }^{19}$ that $P(C \mid G)$ is equivalent to the Boltzmann distribution of an Ising model with constant interaction strength

$$
\mathbb{P}(C \mid G) \propto e^{\beta \sum_{i j} \delta\left(C_{i}, C_{j}\right)}
$$

with inverse temperature $\beta=\log \left(p_{\text {in }} / p_{\text {out }}\right) \approx p_{\text {in }}-p_{\text {out }}$. Note that one of the equilibrium states is trivial assigning all nodes to one cluster. However, asymptotically $(n \rightarrow \infty)$ the probability of this state vanishes and the Boltzmann distribution is uniform over all other configurations with group sizes $n / 2$ and $p_{\text {out }} n / 2$ between-edges ${ }^{19}$. The fact that one of these states is the ground truth partition, and all equilibrium states of Eq. (7) are equivalent up to a permutation of nodes within clusters means they are indistinguishable from the ground truth partition.

Due to the equivalence between Eq. (6) and (7), to prove the equivalence between Eq. (5) and (6) we show that Eq. (5) can also be reduced to Eq. (7). The main insight is that the dynamical OR curvature (Eq. (2)) is constructed using pairs of diffusions, as opposed to single diffusions. Thus, eigenmodes arising from random fluctuations are reflected equally in the spectrum of both diffusions and cancel out upon taking differences over all adjacent node pairs. This allows recovering the community eigenvector $\phi_{c}$ even in the sparse regime where when there is no spectral gap and $\lambda_{c}$ is no longer identifiable from the spectrum (Fig. 2d). Specifically, using pairs of diffusions, we use the spectral expansion to write $\sum_{i j}\left(p_{i}^{u}(\tau)-p_{j}^{u}(\tau)\right)=\sum_{s} e^{\lambda_{s} \tau} \phi_{s} \Delta \phi_{s}$ where

$$
\Delta \phi_{s}:=\sum_{i j}\left(\phi_{s}(i)-\phi_{s}(j)\right)
$$

We find that, on a single SBM realisation, $\Delta \phi_{s}$ is large for only a few eigenvectors $\phi_{s}$ and diminishing for others Fig. 3a). Importantly only those eigenvectors with large $\Delta \phi_{s}$ correlate strongly with the ground truth (Fig. 3a inset). As seen in Fig. 3b, the best eigenvector is not $\phi_{2}$, i.e., the one whose eigenvalue is second in the spectrum and is used by spectral clustering methods, but the one whose eigenvalue is inside the bulk in Fig. 2d and thus cannot be identified by looking at the spectrum alone. The correlation with the ground truth for $\phi_{c}$ with the highest $\Delta \phi_{s}$ averaged over $50 \mathrm{SBM}$ realisations remains close to the highest achievable among all eigenvectors as the KS bound is approached. Meanwhile, $\phi_{2}$ is suboptimal (Fig. 3c). We also found that, close to the KS bound, often a few other eigenvectors with similarly high $\Delta \phi_{s}$ appear, suggesting an improved clustering method combining several top eigenvectors, but this is out of scope here.

To express the curvature in the exponent of Eq. (5) we use the dual formulation of the optimal transport distance (Eq. (14) in Methods). The fact that $\Delta \phi_{c}$ dominates the contribution from other eigenvectors, allows us to approximate $\sum_{i j}\left(p_{i}^{u}(\tau)-p_{j}^{u}(\tau)\right)=e^{\lambda_{c} \tau} \phi_{c} \Delta \phi_{c}+\epsilon_{\phi} \propto e^{\lambda_{c} \tau} \phi_{c}+\epsilon_{\phi}$, where $\epsilon_{\phi}$ is an asymptotically small term. We use this expression, together with the duality formula (Eq. (14)) to express Eq. (5). Finally, in the sparse regime, we may make a tree-like approximation of the neighbourhoods of $i$ and $j$ to find that Eq. (5) reduces to

$$
\mathbb{P}(C \mid \boldsymbol{\kappa}) \propto e^{\left|p_{\text {in }}-p_{\text {out }}\right| \sum_{i j} \delta\left(C_{i}, C_{j}\right)} .
$$


We refer the reader to the Methods for details. Eq. (9) is the same as Eq. (7) when the communities are assortative $\left(p_{\text {in }}>p_{\text {out }}\right)$. We then conclude that the curvatures encode the communities of the symmetric SBM and allow it to be recovered until close to the Kesten-Stigum bound.

In the next section, we present a clustering algorithm based on this insight that can find multiscale clusters in real-world networks.

\section{Geometric modularity for the multiscale clustering of networks}

To exploit the property of the dynamical OR curvature to give multiple geometric representations, we develop a multiscale graph clustering algorithm for real-world networks. Using Eq. (5), we introduce the geometric modularity function

$$
Q_{\kappa}(C, \tau)=\frac{1}{2 m_{\kappa}} \sum_{i j}\left(\kappa_{i j}(\tau)-\kappa_{0}\right) \delta\left(C_{i}, C_{j}\right),
$$

where $2 m_{\kappa}=\sum_{i j}\left|\kappa_{i j}\right|$ is a normalisation factor and $\kappa_{0}=\max _{i j} \kappa_{i j}\left(\tau_{\min }\right)$ is a constant ensuring that all edges have small non-positive curvature at the smallest computed scale $\tau_{\min }$. Hence optimising Eq. (10) at small times yields separate communities for each node whereas at large times, when $\kappa_{i j}(\tau) \rightarrow 1$ for all $i j$, all nodes are merged to a single community. At intermediate scales, the curvatures will have negative and positive values on different edges, making the detection of non-trivial clusters possible without a statistical null-model. This is in contrast to classical modularity ${ }^{35}$, which minimises the expected number of edges between clusters, and requires a statistical null-model (typically the configuration model), which can hinder identifying functional communities based on dynamics ${ }^{5}$.

To detect robust partitions at several scales, we sample the cluster landscape $Q_{\kappa}(C, \tau)$ at a sequence of scales $\tau$ spanning the entire dynamical range of the curvature and, at each $\tau$, optimising Eq. (10) using the Louvain algorithm ${ }^{36,37}$ with 200 random initialisations. At a given $\tau$, we take the cluster with the highest geometric modularity and deem it robust if it has a low variation of information $\mathrm{VI}_{\tau}$ against 50 other randomly chosen clusters at this scale, as well as low variation of information $\mathrm{VI}_{\tau \tau^{\prime}}$ against the best cluster assignments at nearby scales $\tau^{\prime}$. As an example, we show in Fig. 4a the result of this computation on our four-partition SBM graph with two hard-coded scales. We clearly see two large plateaus with low $\mathrm{VI}_{\tau}$ and $\mathrm{VI}_{\tau \tau^{\prime}}$, corresponding to robust clusters, shown in Fig. 4b,c. At the smallest scales we find no robust communities shown by the sharp increase in the number of communities and the large $\mathrm{VI}_{\tau}$.

Due to the link between high edge curvature and well-mixed state (Eq. (3)), we expected that at robust scales the clusters will correspond to those regions which have a high amount of redundant information, and thus can be disconnected without affecting the dynamics within them. To see this, we applied this clustering algorithm to the European power grid graph in Fig. 4d,e,f, an unweighted network of major electrical lines, which has been previously analysed for robustness ${ }^{38}$, multiscale communities ${ }^{39}$ and centrality ${ }^{24}$. The multiscale community structure can be clearly seen with the many minima of the $\mathrm{VI}_{\tau}$ function in Fig. $4 \mathrm{~d}$. We displayed two scales in Fig. 4e,f which unfold parts of the power grid which have been historically independently developed. The smaller scale (at around $\log \tau=-0.95$ ) marks countries or economical and historical alliances (Skandinavia, Benelux, Czechoslovakia, Balkans, etc.). Likewise, the larger scale (at around $\log \tau=-0.5$ ) divides historical Eastern-Western Europe. Interestingly with the boundary in Germany runs along the iron curtain, which also demarcates the regions between major electricity companies.

Finally, we analysed a recent dataset of homeobox gene expression in single neurons of $C$. elegans in Fig. $4 \mathrm{~g}, \mathrm{~h}, \mathrm{i}$ and $\mathrm{j}^{40}$. This work found based on a multivariate linear regression that the homeobox gene expression profile in a given anatomical neuron class can explain on average $74 \%$ of the expression level of the remaining genes in that neuron class. We therefore asked whether the homeobox gene expression profile has sufficient information to cluster neurons into their known anatomical classes.

The data contains a binary feature vector for each of the 301 neurons, indicating the presence of a protein expressed by any of the 105 homeobox genes in the given neuron. To convert this data into a 
a

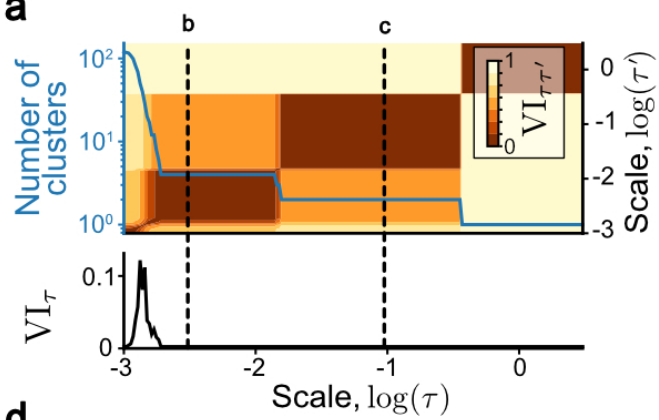

d
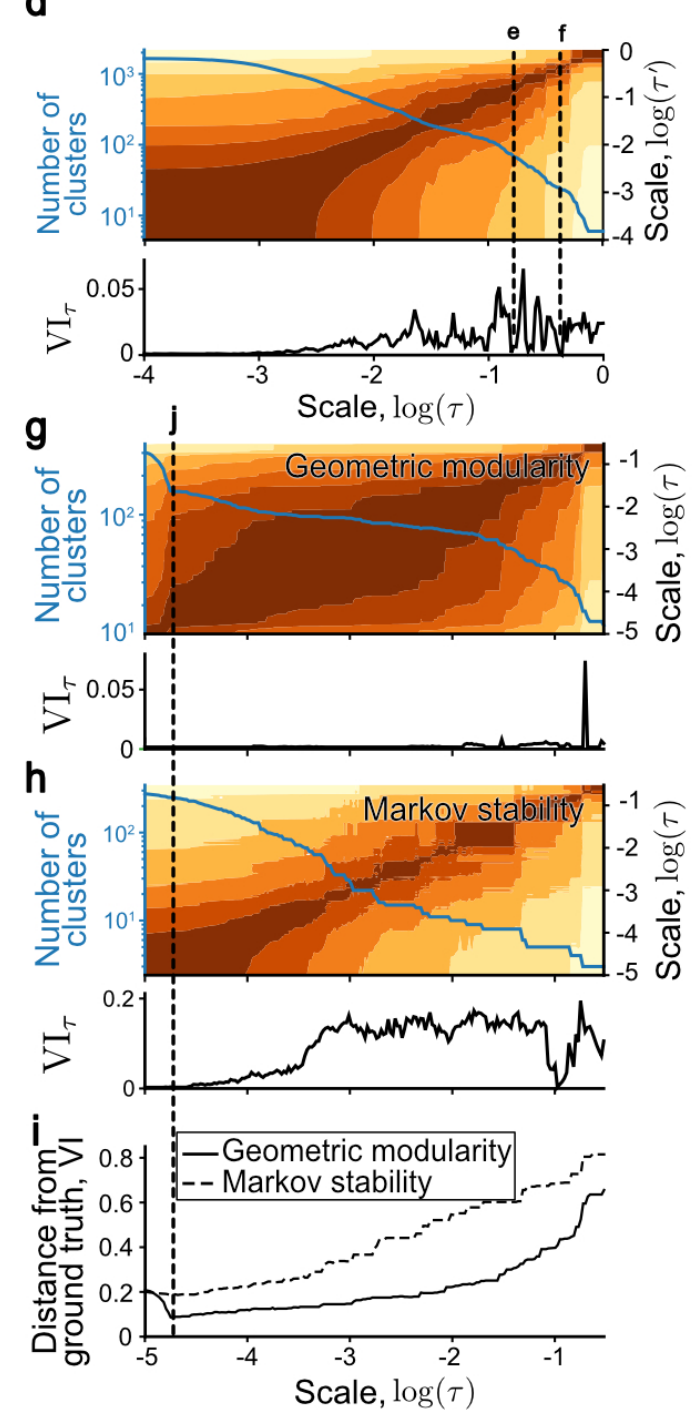

b

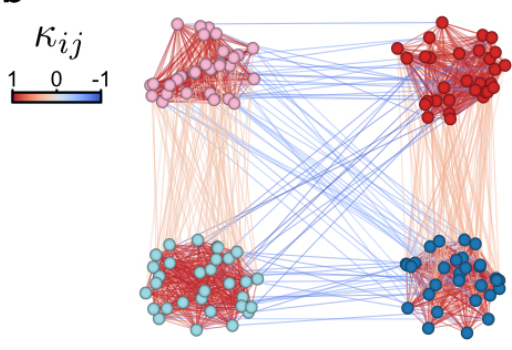

C

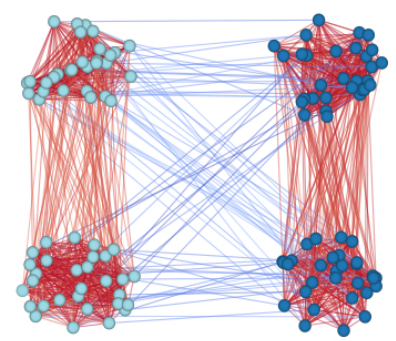

e

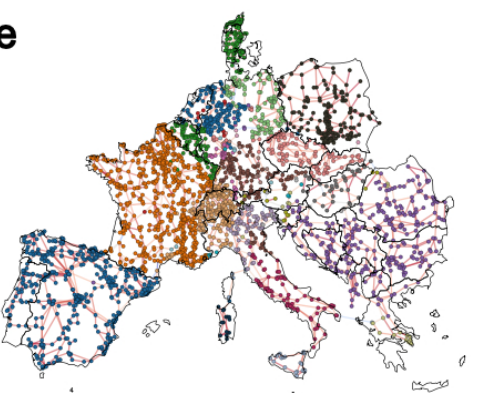

f
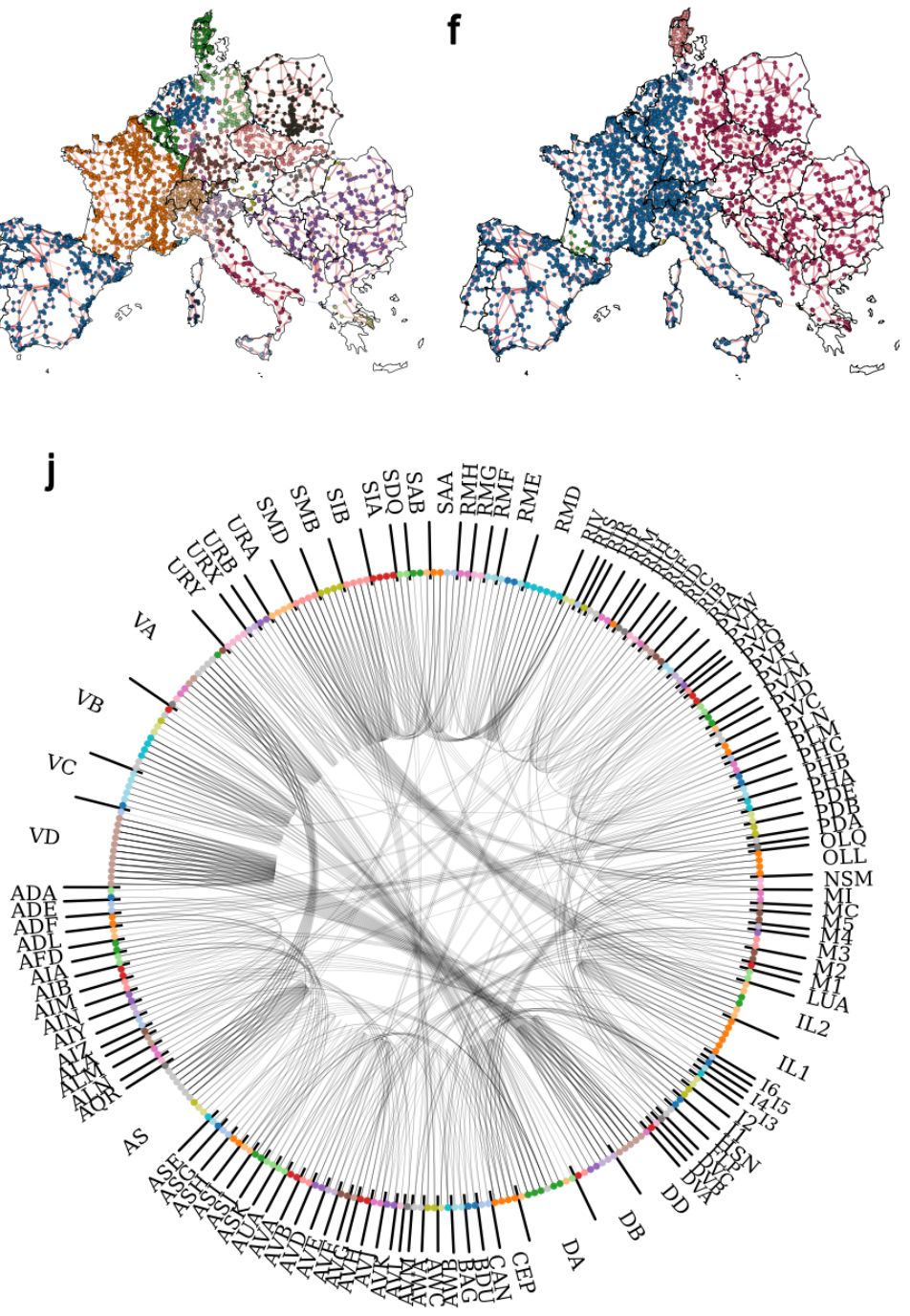

Figure 4: Clustering networks based on multiscale geometric modularity a Clustering statistics computed based on $10^{2}$ Louvain realisations for the multiscale stochastic block model graph. Vertical dashed lines show scales at which stable clusters are detected based on low variation of information at a given scale and persistent low variation of information between Louvain realisations across scales. The communities obtained at these scales are shown on $\mathbf{b}$ for $\log \tau=-2.3$ and $\mathbf{c}$ for $\log \tau=-1$. Edges are coloured by the curvatures at the respective scales. $\mathbf{d}$ Clustering statistics for the European power grid. Two representative stable scales are shown $\mathbf{e}$ for $\log \tau=-0.95$ and $\mathbf{f}$ for $\log \tau=-0.5$. g Clustering statistics for and the network of C. elegans single-neuron homeobox gene expressions show a plateau of stable scales with very similar partitions. h Clustering statistics obtained with Markov stability shows stable scales only at small times with single-node communities, indicating overfitting, and many non-robust partitions at larger scales with high variation of information. i Distance from ground truth based on structural neuronal types or the predicted clusters. Geometric modularity obtained significantly better performance than Markov stability. j Clustering of the C. elegans homeobox gene expression data obtained from geometric modularity optimisation superimposed with the ground truth. 
graph with nodes being neurons, we first eliminated all homeobox genes co-expressed in none or more than $90 \%$ of the neurons to retain 67 homeobox genes. We then constructed an all-to-all graph adjacency matrix weighted by the Jaccard similarity index between expression profiles of neurons. To increase the number of edges with negative curvature, thus improve the detection at the smallest scales, we sparsified this network using a geometric sparsification method ${ }^{41}$ with parameter $\gamma=0.01$. This method retains at most a fraction $\gamma$ edges of the original graph as minimum spanning tree augmented by edges relevant for preserving local or global geometry of the graph.

The results of our clustering algorithm on this graph is shown on Fig. $4 \mathrm{~g}$ and compared with the result of Markov stability ${ }^{8}$ (Fig. 4h), a multiscale method based on persistence of diffusions. Geometric modularity obtains a large range of robust scales with highly similar clusters - as shown by the low $\mathrm{VI}_{\tau}$ and $\mathrm{VI}_{\tau \tau^{\prime}}$. These scales correlate closely with the known ground truth of 117 anatomical neuron classes (Fig. 4i). In contrast, for Markov stability ${ }^{8}$, the scales with low $V I_{\tau}$ overfit the graph finding too many clusters (Fig. 4h) which correlate less with the ground truth (Fig. 4i). Likewise, hierarchical clustering fails to identify the ground truth communities ${ }^{40}$. On Fig. $4 \mathrm{j}$ we superimpose the best clustering from geometric modularity against the ground-truth. We observe little differences, apart from VA and AS nodes as well as VD and DD often clustered together. Careful look reveals close biological relationship between these classes; all four classes correspond to motor neurons, with pairs expressing the same neurotransmitters - VA, AS expressing acetylcholine and VD, DD expressing gamma-aminobutyric acid (GABA). These novel results give direct quantitative support to the claim that homeobox gene expression patterns encode structural neuron types. We also observe other stable partitions at larger scale, but they did not correlate the ground-truth.

Overall, these results give a strong demonstration that our method is able to find stable clusters in sparse graphs, and provide meaningful insights into distinct types of real-world networks.

\section{Discussion}

We introduced the concept of dynamical Ollivier-Ricci (OR) curvature which defines an effective geometry from pairs of diffusion processes on the network. Instead of imposing the requirement of a manifold approximation or embedding, used by previous geometric approaches ${ }^{4-6,23}$, our approach constructs a geometric object - the weighted and signed edge curvature matrix - capturing progressively coarser features as the diffusion processes evolve.

Real-world networks often exhibit community structure on multiple scales, based on difference between the rates of information propagation in regions the network on various timescales. We showed that the edge curvature matrix carries a precise meaning in this context and bounds the rate of information flow across edges. Consequentially, curvature gaps, differences between edge curvatures within and between regions, indicate network bottlenecks. This result does not rely on the dynamics being linear diffusions, making it suitable to study the interaction of arbitrary dynamical processes. We expect that, in the future, this approach can be used to tune the geometry of the graph to control the flux or interaction of network-driven dynamical processes, for example, leading to better insights to synchronisation problems or metapopulation models $^{42}$.

Although diffusion processes constructed from the graph Laplacian have been explored for network clustering $^{8,23}$, our work differs in the use of diffusion pairs to construct the curvature. Two diffusions pick up random variations in the graph independently, which can be exploited to average out non-informative fluctuations. On stochastic block models, this feature allows the curvature gap to robustly indicate clusters in the sparse regime down to the fundamental limit, where clustering methods relying on the spectral gap in the Laplacian fail ${ }^{32}$. We also found a new measure of eigenvalue quality, able to select the best eigenvector to be used in spectral methods. Interestingly, the edge curvatures are defined on the set of shortest paths which cannot contain the same edge twice, a subset of the set of non-backtracking walks. Our results are therefore consistent with previous works on the limits of cluster detection using statistical physics objects including the spectrum of non-backtracking operator ${ }^{21}$ or related message passing approaches ${ }^{19}$. We expect this insight to provide a new avenue to study the fundamental limits of efficient clustering from 
a geometric perspective.

Finally, using geometric modularity we built an easy-to-use algorithm to study the multiscale community structure of real networks. We demonstrated on the European power grid network and a recent dataset of $C$. elegans single-neuron homeobox gene expressions that our method can find robust and interpretable communities on multiple scales on diverse datasets without the tendency of overfitting.

Overall, we expect our insights connecting dynamical processes, geometry and network clustering to open new avenues to studying and controlling the structural and dynamical properties of networks.

\section{Acknowledgements}

AG acknowledges support from an HFSP Cross-disciplinary Postdoctoral Fellowship (LT000669/2020-C). We thank Mauricio Barahona for insightful discussions on this topic, Jonas Braun and István Tomon for their helpful comments on the manuscript and Daniel Morales for inspiring us to analyse the C. elegans dataset.

\section{Author contributions}

A.G. and A.A. contributed equally to this work.

\section{Code availability}

The code to reproduce the results in our paper and to perform geometric modularity optimisation is available at https://github.com/agosztolai/geometric_clustering.

\section{Data availability}

The raw data supporting the results is available from the authors upon request.

\section{Methods}

\section{Classical Ollivier-Ricci edge curvature}

To contrast the dynamical Ollivier Ricci curvature in Eq. (2), we recap the definition of the classical formulation ${ }^{9}$, which is a generalisation of the Ricci curvature of manifolds in differential geometry. Briefly, consider two close points $x$ and $y$ on a manifold as well as a vector $\mathbf{v}$ on the tangent plane at $x$ and another tangent vector $\mathbf{v}^{\prime}$ in the tangent plane at $y$ that is parallel to $\mathbf{v}$, i.e., obtained by parallel transport along the geodesic connecting $x, y$ (Supplementary Figure 1a). These vectors shift $x$ and $y$ to nearby points $x^{\prime}$ and $y^{\prime}$, which will be at a distance $d_{x^{\prime} y^{\prime}} \approx d_{x y}\left(1-\|\mathbf{v}\|^{2} K_{w} / 2\right)$, where $K_{w}$ is the sectional curvature. The Ricci curvature $\operatorname{Ric}_{x y}$ between points $x, y$ is then defined as the average sectional curvature and is proportional to

$$
R i c_{x y} \propto 1-\frac{\left\langle d_{x^{\prime} y^{\prime}}\right\rangle}{d_{x y}}
$$

where $\langle\cdot\rangle$ denotes the average over all vectors $\mathbf{w}, \mathbf{w}^{\prime}$ running over the unit sphere in the tangent planes at $x$ and $y$. In other words, it measures how much geodesics expand or contract on average around points $x, y$. On flat planes the geodesics stay equally separated hence $R i c_{x y}=0$, on spheres the geodesics contract hence $R i c_{x y}>0$, whereas in hyperbolic spaces they expand, hence $R i c_{x y}<0$ (Supplementary Figure 1a).

The classical Ollivier-Ricci curvature ${ }^{9}$ is defined by direct analogy to this. Consider two adjacent nodes $i$ and $j$ and place weights on their immediate neighbours in proportion to the edge weights, namely, $\mathbf{p}_{i}=\delta_{i} \mathbf{K}^{-1} \mathbf{A}$. Then the Ollivier Ricci curvature becomes

$$
\kappa_{i j}=1-\frac{\mathcal{W}_{1}\left(\mathbf{p}_{i}, \mathbf{p}_{j}\right)}{d_{i j}}
$$

Contrast this expression to the dynamical Ollivier Ricci curvature in Eq. (2), which considers diffusion measures which weight progressively larger neighbourhoods as $\tau$ increases.

We remark that Eq. (12) and the dynamical OR curvature in (2) are valid for any two nodes $i, j$ if the denominator is replaced by the weighted geodesic distance between $i$ and $j$, but for this work, it suffices to consider adjacent nodes. Indeed, for any non-adjacent nodes $u v, \kappa_{u v} \geq \kappa_{u^{\prime} v^{\prime}}$, where $u^{\prime} v^{\prime}$ is an adjacent pair lying on the geodesic connecting $u, v$ (Proposition 19 in Ref. ${ }^{9}$ ), meaning that local curvatures control global curvatures. 


\section{Wasserstein distance}

To measure the distance between a pair of measures $\mathbf{p}_{i}(\tau)$ and $\mathbf{p}_{j}(\tau)$ we use the optimal transport distance ${ }^{29}$ (also known as 1-Wasserstein or earth-mover distance), defined as

$$
\begin{aligned}
& \mathcal{W}_{1}\left(\mathbf{p}_{i}(\tau), \mathbf{p}_{j}(\tau)\right)=\min _{\zeta} \sum_{u v} d_{u v} \zeta_{u v}, \\
& \text { subject to } \sum_{v} \zeta_{u v}=p_{i}^{u}(\tau), \quad \sum_{u} \zeta_{u v}=p_{j}^{v}(\tau) .
\end{aligned}
$$

The constraints in Eq. (13) ensure that the optimal transport plan $\boldsymbol{\zeta}(\tau) \in \mathbb{R}^{n \times n}$ is a coupling of the measures $\mathbf{p}_{i}(\tau), \mathbf{p}_{j}(\tau)$, i.e., $\boldsymbol{\zeta}(\tau)$ is a joint distribution that admits $\mathbf{p}_{i}(\tau)$ and $\mathbf{p}_{j}(\tau)$ as marginals.

An equivalent formulation of this distance can be constructed from the Kantorovich-Rubinstein duality ${ }^{29}$, given by

$$
\mathcal{W}_{1}\left(\mathbf{p}_{i}(\tau), \mathbf{p}_{j}(\tau)\right)=\sup _{f} \sum_{u} f(u)\left[p_{i}^{u}(\tau)-p_{j}^{u}(\tau)\right]
$$

where the supremum is taken over all 1-Lipschitz functions $f$ on the graph, that is,

$$
|f(u)-f(v)| \leq d_{u v}
$$

for any node pair $u, v$.

\section{Computational complexity}

The computational complexity of our clustering method is determined by three components: the computation of the diffusion measures (Eq. (1)), the computation of the optimal transport distance (Eq. (13)) and the computation of the clustering.

We compute each diffusion measure in Eq. (1) by the scaling and squaring algorithm of Ref. ${ }^{43}$. To our knowledge, the complexity of this algorithm is not known, but we found it to be better than computing the matrix exponential which runs in time $\mathcal{O}\left(n^{3}\right)$ and then multiplying by the initial condition. The exact computation of Eq. (13) is performed by interior point methods which have a complexity $\mathcal{O}\left(n^{3} \log n\right)$. However, note that in our work the explicit computation of $\boldsymbol{\zeta}(\tau)$ is not required and, moreover, there is typically a significant overlap between the measures $\mathbf{p}_{i}, \mathbf{p}_{j}$ whenever $i, j$ lie in the same highly connected region. Based on these observations we use recent approximate algorithms to compute the transport cost permitting near $\mathcal{O}(n)$-time computation of the optimal transport distance ${ }^{44,45}$. This yields a computational complexity of $\mathcal{O}(n m)$ for sparse graphs with worst case $\mathcal{O}\left(n^{3}\right)$. These methods also allow GPU parallelisation, which we recommend using for large $\left(n \gg 10^{3}\right)$ and dense graphs.

The third component is the complexity of the Louvain algorithm which is linear $\mathcal{O}(n)$ for sparse networks ${ }^{36}$.

\section{Upper bound on the mixing time in terms of curvature}

Here we prove inequality (3), which gives an upper bound on the mixing time of the coupled diffusions with measures $\mathbf{p}_{i}(\tau)$, $\mathbf{p}_{j}(\tau)$ in terms the dynamical OR curvature. The $\epsilon$-mixing time is defined as the smallest $\tau$ where the law of the coupled process, the optimal transport plan $\boldsymbol{\zeta}(\tau)$, is within an $\epsilon$ radius of the stationary distribution

$$
\tau_{i j}(\epsilon):=\min \left\{\tau:\|\boldsymbol{\zeta}(\tau)-\boldsymbol{\zeta}(\infty)\|_{\mathrm{TV}} \leq \epsilon\right\},
$$

where the notion of "close to stationarity" is quantified by the total variation distance $\|\boldsymbol{\zeta}(\tau)-\boldsymbol{\zeta}(\infty)\|_{\mathrm{TV}}:=\frac{1}{2} \sum_{u v} \mid \zeta_{u v}(\tau)-$ $\zeta_{u v}(\infty) \mid$. Since $\mathbf{p}_{i}(\tau)$ and $\mathbf{p}_{j}(\tau)$ are marginals of $\boldsymbol{\zeta}(\tau)$ we have that

$$
\begin{aligned}
\tau_{i j}(\epsilon) & =\min \left\{\tau:\left\|\mathbf{p}_{i}(\tau)-\boldsymbol{\pi}\right\|_{\mathrm{TV}}+\left\|\mathbf{p}_{j}(\tau)-\boldsymbol{\pi}\right\|_{\mathrm{TV}} \leq \epsilon\right\} \\
& =\min \left\{\tau:\left\|\mathbf{p}_{i}(\tau)-\mathbf{p}_{j}(\tau)\right\|_{\mathrm{TV}} \leq \epsilon\right\}
\end{aligned}
$$

where we used the independence of the diffusion processes. From here, we may follow Ref. ${ }^{46}$ and use the Csiszár-KullbackPinsker inequality for the optimal transport distance

$$
\left\|\mathbf{p}_{j}(\tau)-\mathbf{p}_{j}(\infty)\right\|_{\mathrm{TV}} \leq\left(1 / d_{0}\right) \mathcal{W}_{1}\left(\mathbf{p}_{j}(\tau), \mathbf{p}_{j}(\tau)\right)
$$

where $d_{0}=\min _{i j} d_{i j}$ is a global graph constant, which can therefore be absorbed into $\epsilon$. This gives an upper bound

$$
\begin{aligned}
\tau_{i j}\left(\epsilon^{\prime}\right) & \leq \min \left\{\tau: \mathcal{W}_{1}\left(\mathbf{p}_{i}(\tau), \mathbf{p}_{j}(\tau)\right) \leq \epsilon^{\prime}\right\} \\
& =\min \left\{\tau: \kappa_{i j}(\tau) \geq 1-\epsilon^{\prime}\right\},
\end{aligned}
$$

with $\epsilon^{\prime}=d_{0} \epsilon$ which is what we set out to show. Note that choosing any $\epsilon^{\prime} \in(0,1 / 2)$ ensures exponential convergence rate to the stationary measure ${ }^{47}$ and by convention, we take the middle of this range and define $\tau_{i j}^{\text {mix }}:=\tau_{i j}^{\text {mix }}(1 / 4)$ to obtain Eq. (3). 


\section{Connection between geometric modularity and the symmetric stochastic block model}

In this section, we prove that the Boltzmann distribution of cluster assignments given the edge curvatures $\mathbb{P}(C \mid \kappa)($ Eq. $(5))$ has equilibrium states which are indistinguishable from the ground truth partition of the SBM. We show this by reducing $\mathbb{P}(C \mid \kappa)$ as well as the posterior distribution $\mathbb{P}(C \mid G)$, to the same constant interaction Ising model (Eq. (7)). In the remainder of this section we work in the sparse regime, where $p_{\text {in }}, p_{\text {out }}=O(1 / n)$.

First, we recap the well-known equivalence of the SBM and the Ising model ${ }^{19}$. Let $E$ denote the set of edges. The probability distribution of the symmetric SBM for two clusters can be written as ${ }^{30}$

$$
\begin{aligned}
\mathbb{P}(G \mid C) & =p_{\text {out }}^{e}\left(1-p_{\text {out }}\right)^{\left(\begin{array}{l}
n \\
2
\end{array}\right)-e} \times \\
& \times \prod_{i j \in E}\left(\frac{p_{\text {in }}}{p_{\text {out }}}\right)^{\delta\left(C_{i}, C_{j}\right)} \prod_{i j \notin E}\left(\frac{1-p_{\text {in }}}{1-p_{\text {out }}}\right)^{\delta\left(C_{i}, C_{j}\right)} \\
& \propto \prod_{i j \in E}\left(\frac{p_{\text {in }}}{p_{\text {out }}}\right)^{\delta\left(C_{i}, C_{j}\right)}
\end{aligned}
$$

where $e$ is the total number of edges and in the last line we used that the effect of non-edges is weak in the sparse regime. Therefore, by Bayes' theorem with uniform prior one obtains the posterior distribution $\mathbb{P}(C \mid G) \propto \mathbb{P}(G \mid C)$. As a result, the probability of clusters generated by the SBM is equivalent to the Ising model with uniform interaction with Boltzmann distribution given by Eq. (7) ${ }^{19}$.

Second, we reduce the Boltzmann distribution of clusters given the edge curvature to same Ising model in Eq. (7). From Eq. (5) we have

$$
\begin{aligned}
\mathbb{P}(C \mid \boldsymbol{\kappa}) & \propto e^{\sum_{i j} \kappa_{i j}(\tau) \delta\left(C_{i}, C_{j}\right)} \\
& \propto e^{\sum_{i j}\left[1-\mathcal{W}_{1}\left(\mathbf{p}_{i}(\tau), \mathbf{p}_{j}(\tau)\right)\right] \delta\left(C_{i}, C_{j}\right)}
\end{aligned}
$$

where in the last line we used the definition of the curvature in Eq. (2). Comparing Eq. (18) with Eq. (7) note that $1-\mathcal{W}_{1}\left(\mathbf{p}_{i}(\tau), \mathbf{p}_{j}(\tau)\right)$ is non-constant and has a non-linear dependence on the scale $\tau$. However, it is possible to express it in terms of $p_{\text {in }}, p_{\text {in }}$ to make the connection to the Ising model. Let us write the diffusion measures in Eq. (1) in terms of the spectral decomposition of $\mathbf{L}$ as

$$
p_{i}^{k}(\tau)=\delta_{i} \sum_{s=1}^{n} e^{-\lambda_{s} \tau} \phi_{s}(k) \phi_{s}(l)=\sum_{s=1}^{n} e^{-\lambda_{s} \tau} \phi_{s}(k) \phi_{s}(i) .
$$

At this point let us remark that in the dense regime where $p_{\text {in }}, p_{\text {out }}=O(1)$, the first two eigenmodes $\left(\lambda_{1}, \phi_{1}\right)$ and $\left(\lambda_{c}, \phi_{c}\right)$ dominate and the second eigenmode contains the anti-symmetric eigenvector $\phi_{c}(u)=1 / \sqrt{n}$ when $C_{u}=1$ and $-1 / \sqrt{n}$ when $C_{u}=2$ that is associated with the community structure (Fig. 2c). Thus, one can follow spectral clustering methods ${ }^{27}$ to find the sparsest cut between clusters using $\phi_{c}$. In contrast, in the sparse regime, the dominant eigenmodes will be driven by random fluctuations in the node degrees across the graph ${ }^{48}$, thus spectral clustering algorithms based on $\mathbf{L}$ are suboptimal (Fig. 2d).

However, the coupled diffusion pair allows for cancelling out random fluctuations in their spectrum. To see this, consider for a between-edge $i j$ the difference

$$
\begin{aligned}
\sum_{i j \in E} p_{i}^{k}(\tau)-p_{j}^{k}(\tau)= & \sum_{i j \in E} \sum_{s=1}^{n} e^{-\lambda_{s} \tau} \phi_{s}(k)\left[\phi_{s}(i)-\phi_{s}(j)\right] \\
& =\sum_{s=1}^{n} e^{-\lambda_{s} \tau} \phi_{s}(k) \Delta \phi_{s}
\end{aligned}
$$

where $\Delta \phi_{s}$ is defined in Eq. (8). The first term involves the constant eigenvector $\phi_{1}$ corresponding to the stationary state. Therefore, $\phi_{1}(i)=\phi_{1}(j)$ for all $i j$ and thus its contributions cancels out when taking differences. Further, for eigenvectors $\phi_{s}$ with $s \neq 1, c$ we have asymptotically $(n \rightarrow \infty)$ that

$$
\Delta \phi_{s} \rightarrow 0
$$

(Fig. 3). As a result, the only contribution we are left with is coming from the anti-symmetric eigenmode $\left(\lambda_{c}, \phi_{c}\right)$. Thus we have that

$$
\sum_{i j \in E}\left(p_{i}^{u}(\tau)-p_{j}^{u}(\tau)\right)=\left\{\begin{array}{l}
\epsilon_{\phi}, \text { if } C_{i}=C_{j}, \\
e^{-\lambda_{c} \tau} \phi_{c} \Delta \phi_{c}+\epsilon_{\phi}, \text { if } C_{i} \neq C_{j},
\end{array}\right.
$$

where $\epsilon_{\phi}$ represents the contribution from the random eigenvectors which is negligible in the limit $n \rightarrow \infty$.

To compute $\mathcal{W}_{1}$ in the exponent of Eq. (18), we use Kantorovich-Rubinstein duality (Eq. (14)). Using Eq. (21) in Eq. (14) and ignoring asymptotically small terms, we consider the quantity

$$
\sum_{i j \in E} \sum_{u} f(u)\left[p_{i}^{u}(\tau)-p_{j}^{u}(\tau)\right]
$$




$$
\begin{aligned}
= & e^{-\lambda_{c} \tau} \sum_{u} f(u) \phi_{c}(u) \\
= & \frac{e^{-\lambda_{c} \tau}}{n}\left[\sum_{u: C_{u}=1} f(u)-\sum_{u: C_{u}=2} f(u)\right] \\
= & \frac{e^{-\lambda_{c} \tau}}{n}\left[\sum_{u: C_{u}=1}(f(u)-f(i))-\sum_{u: C_{u}=2}(f(u)-f(j))\right. \\
& \left.\quad+\sum_{u: C_{u}=1} f(i)-\sum_{u: C_{u}=2} f(j)\right] .
\end{aligned}
$$

In the sparse regime, we may make a tree-like approximation in the neighbourhood of $i$. This means that the number of neighbours of $i$ at distance $q$ inside the cluster is $p_{\text {in }}^{q}(n / 2)^{q}$, ignoring terms of order $O(1 / n)$ and beyond. Considering only nodes at unit distance $(q=1)$, we approximate Eq. (22) as

$$
\begin{aligned}
& \frac{e^{-\lambda_{c} \tau}}{n}\left[\sum_{\substack{u: C_{u}=1 \\
u \sim i}}(f(u)-f(i))-\sum_{\substack{u: C_{u}=2 \\
u \sim i}}(f(u)-f(i))\right. \\
& \quad+\sum_{\substack{u: C_{u}=1 \\
u \sim j}}(f(u)-f(j))-\sum_{\substack{u: C_{u}=2 \\
u \sim j}}(f(u)-f(j)) \\
& \left.\quad+\sum_{\substack{u: C_{u}=1 \\
u \sim i}} f(i)-\sum_{\substack{u: C_{u}=2 \\
u \sim i}} f(i)+\sum_{\substack{u: C_{u}=1 \\
u \sim j}} f(j)-\sum_{\substack{u: C_{u}=2 \\
u \sim j}} f(j)\right] \\
& =\frac{e^{-\lambda_{c} \tau}}{n}\left[\sum_{\substack{u: C_{u}=1 \\
u \sim i}}(f(u)-f(i))-\sum_{\substack{u: C_{u}=2 \\
u \sim i}}(f(u)-f(i))\right. \\
& \quad+\sum_{\substack{u: C_{u}=1 \\
u \sim j}}(f(u)-f(j))-\sum_{\substack{u: C_{u}=2 \\
u \sim j}}(f(u)-f(j)) \\
& \left.\quad+\frac{n}{2} p_{\text {in }}(f(i)-f(j))-\frac{n}{2} p_{\text {out }}(f(i)-f(j))\right] .
\end{aligned}
$$

Then, taking the supremum over all 1-Lipschitz functions $f$, we obtain

$$
\begin{aligned}
& \sum_{i j \in E} \mathcal{W}_{1}\left(\mathbf{p}_{i}(\tau), \mathbf{p}_{j}(\tau)\right)\left(1-\delta\left(C_{i}, C_{j}\right)\right) \\
& \approx e^{-\lambda_{c} \tau}\left(p_{\text {in }}+p_{\text {out }}\right)\left(1+\frac{\left|p_{\text {in }}-p_{\text {out }}\right|}{2\left(p_{\text {in }}+p_{\text {out }}\right)}\right)
\end{aligned}
$$

Substituting this into Eq. (18) and noting that $p_{\text {in }}+p_{\text {out }}$ is constant we obtain at a fixed $\tau$

$$
\mathbb{P}(C \mid \boldsymbol{\kappa}) \propto \exp \left[\left(\frac{\left|p_{\text {in }}-p_{\text {out }}\right|}{2\left(p_{\text {in }}+p_{\text {out }}\right)}\right) \sum_{i j \in E} \delta\left(C_{i}, C_{j}\right)\right],
$$

which up to a constant of proportionality equals the expression in Eq. (9).

\section{References}

[1] Tenenbaum, J. B., de Silva, V. \& Langford, J. C. A global geometric framework for nonlinear dimensionality reduction. Science 290, 2319-2323 (2000).

[2] Ding, C., Xiaofeng He, Hongyuan Zha \& Simon, H. D. Adaptive dimension reduction for clustering high dimensional data. In 2002 IEEE International Conference on Data Mining, 2002. Proceedings., 147-154 (2002).

[3] Serrano, M. A., Krioukov, D. \& Boguñá, M. Self-similarity of complex networks and hidden metric spaces. Phys. Rev. Lett. 100, 078701 (2008).

[4] García-Pérez, G., Boguñá, M. \& Serrano, M. Á. Multiscale unfolding of real networks by geometric renormalization. Nat. Phys. 1-9 (2018).

[5] De Domenico, M. Diffusion geometry unravels the emergence of functional clusters in collective phenomena. Phys. Rev. Lett. 118, 168301 (2017). 
[6] Brockmann, D. \& Helbing, D. The hidden geometry of complex, network-driven contagion phenomena. Science 342, $1337-1342$ (2013).

[7] Matousek, J. Lectures on discrete geometry, vol. 212 (Springer New York, 2013).

[8] Delvenne, J.-C., Yaliraki, S. N. \& Barahona, M. Stability of graph communities across time scales. Proc. Natl. Acad. Sci. U.S.A. 107, 12755-12760 (2010).

[9] Ollivier, Y. Ricci curvature of Markov chains on metric spaces. J. Funct. Anal. 256, 810-864 (2009).

[10] Sturm, K.-T. On the geometry of metric measure spaces. Acta Math. 196, 65-131 (2006).

[11] Lott, J. \& Villani, C. Ricci curvature for metric-measure spaces via optimal transport. Ann. Math. 169, 903-991 (2009).

[12] van der Hoorn, P., Cunningham, W. J., Lippner, G., Trugenberger, C. \& Krioukov, D. Ollivier-Ricci curvature convergence in random geometric graphs. Preprint at https://arxiv.org/abs/2008.01209 (2020).

[13] Jost, J. \& Liu, S. Ollivier's ricci curvature, local clustering and curvature-dimension inequalities on graphs. Discrete Ef Comput. Geom. 51, 300-322 (2014).

[14] Bauer, F., Jost, J. \& Liu, S. Ollivier-Ricci curvature and the spectrum of the normalized graph Laplace operator. Math. Res. Lett. 19, 1185-1205 (2012).

[15] Sandhu, R. S., Georgiou, T. T. \& Tannenbaum, A. R. Ricci curvature: An economic indicator for market fragility and systemic risk. Sci. Adv. 2 (2016).

[16] Farooq, H., Chen, Y., Georgiou, T. T., Tannenbaum, A. \& Lenglet, C. Network curvature as a hallmark of brain structural connectivity. Nat. Commun. 10, 4937 (2019).

[17] Sia, J., Jonckheere, E. \& Bogdan, P. Ollivier-Ricci curvature-based method to community detection in complex networks. Sci. Rep. 9, 9800 (2019).

[18] Ni, C.-C., Lin, Y.-Y., Luo, F. \& Gao, J. Community detection on networks with Ricci flow. Sci. Rep. 9, 9984 (2019).

[19] Decelle, A., Krzakala, F., Moore, C. \& Zdeborová, L. Asymptotic analysis of the stochastic block model for modular networks and its algorithmic applications. Phys. Rev. E 84, 066106 (2011).

[20] Abbé, E. \& Sandon, C. Community detection in general stochastic block models: Fundamental limits and efficient algorithms for recovery. In 2015 IEEE 56th Annual Symposium on Foundations of Computer Science, 670-688 (2015).

[21] Massoulié, L. Community detection thresholds and the weak Ramanujan property. In Proceedings of the Forty-Sixth Annual ACM Symposium on Theory of Computing, STOC '14, 694-703 (Association for Computing Machinery, New York, NY, USA, 2014).

[22] Gfeller, D. \& De Los Rios, P. Spectral coarse graining of complex networks. Phys. Rev. Lett. 99, 038701 (2007).

[23] Coifman, R. R. et al. Geometric diffusions as a tool for harmonic analysis and structure definition of data: Diffusion maps. Proc. Natl. Acad. Sci. U.S.A. 102, 7426-7431 (2005).

[24] Arnaudon, A., Peach, R. L. \& Barahona, M. Scale-dependent measure of network centrality from diffusion dynamics. Phys. Rev. Res. 2, 033104 (2020).

[25] Schaub, M. T., Billeh, Y. N., Anastassiou, C. A., Koch, C. \& Barahona, M. Emergence of slow-switching assemblies in structured neuronal networks. PLoS Comp Biol 11, e1004196-28 (2015).

[26] Young, H. P. Innovation diffusion in heterogeneous populations: Contagion, social influence, and social learning. Am. Econ. Rev. 99, 1899-1924 (2009).

[27] Chung, F. R. K. Spectral Graph Theory, vol. 92 (American Mathematical Soc., 1997).

[28] Veysseire, L. Coarse Ricci curvature for continuous-time Markov processes. Preprint at https://arxiv.org/abs/1202.0420 (2012).

[29] Villani, C. Optimal transport: old and new (Springer, Berlin Heidelberg, 2009).

[30] Holland, P. W., Laskey, K. B. \& Leinhardt, S. Stochastic blockmodels: First steps. Soc. Netw. 5, 109-137 (1983). 
[31] Gosztolai, A., Carrillo, J. A. \& Barahona, M. Collective search with finite perception: Transient dynamics and search efficiency. Front. Phys. 6, 153 (2019).

[32] Kawamoto, T. \& Kabashima, Y. Limitations in the spectral method for graph partitioning: Detectability threshold and localization of eigenvectors. Phys. Rev. E 91, 062803 (2015).

[33] Kay, S. M. Fundamentals of statistical signal processing. Prentice Hall signal processing series (Prentice Hall PTR, Upper Saddle River, NJ, 1993).

[34] Mossel, E., Neeman, J. \& Sly, A. A proof of the block model threshold conjecture. Combinatorica 38, 665-708 (2018).

[35] Newman, M. E. J. Modularity and community structure in networks. Proc. Natl. Acad. Sci. U.S.A. 103, 8577-8582 (2006).

[36] Blondel, V. D., Guillaume, J.-L., Lambiotte, R. \& Lefebvre, E. Fast unfolding of communities in large networks. J. Stat. Mech. Theory Exp. 2008, P10008 (2008).

[37] PyGenStability: unsupervised clustering with generalised Louvain and Markov Stability. In preparation (2021).

[38] Rosas-Casals, M., Valverde, S. \& Solé, R. V. Topological vulnerability of the European power grid under errors and attacs. Int. J. Bifurcat. Chaos 17, 2465-2475 (2007).

[39] Schaub, M. T., Delvenne, J.-C., Yaliraki, S. N. \& Barahona, M. Markov dynamics as a zooming lens for multiscale community detection: Non clique-like communities and the field-of-view limit. PLOS One 7, 1-11 (2012).

[40] Reilly, M. B., Cros, C., Varol, E., Yemini, E. \& Hobert, O. Unique homeobox codes delineate all the neuron classes of $C$. elegans. Nature 584, 595-601 (2020).

[41] Beguerisse-Diaz, M., Vangelov, B. \& Barahona, M. Finding role communities in directed networks using role-based similarity, markov stability and the relaxed minimum spanning tree. 2013 IEEE Global Conference on Signal and Information Processing (2013).

[42] Davis, J. T., Perra, N., Zhang, Q., Moreno, Y. \& Vespignani, A. Phase transitions in information spreading on structured populations. Nat. Phys. 1-8 (2020).

[43] Al-Mohy, A. H. \& Higham, N. J. A new scaling and squaring algorithm for the matrix exponential. SIAM J. Matrix Anal. Appl. 31, 970-989 (2010).

[44] Cuturi, M. Sinkhorn distances: Lightspeed computation of optimal transport. In Burges, C. J. C., Bottou, L., Welling, M., Ghahramani, Z. \& Weinberger, K. Q. (eds.) Adv. Neural. Inf.. Process. Syst. 26, 2292-2300 (Curran Associates, Inc., 2013).

[45] Atasu, K. \& Mittelholzer, T. Linear-complexity data-parallel earth mover's distance approximations. In Chaudhuri, K. \& Salakhutdinov, R. (eds.) Proceedings of the 36th International Conference on Machine Learning, vol. 97 of Proceedings of Machine Learning Research, 364-373 (PMLR, Long Beach, California, USA, 2019).

[46] Paulin, D. Mixing and concentration by Ricci curvature. J. Func. Anal. 270, 1623-1662 (2016).

[47] Levin, D. A., Peres, Y. \& Wilmer, E. L. Markov chains and mixing times (American Mathematical Society, 2006).

[48] Krivelevich, M. \& Sudakov, B. The largest eigenvalue of sparse random graphs. Preprint at https://arxiv.org/math/0106066v1 (2001). 

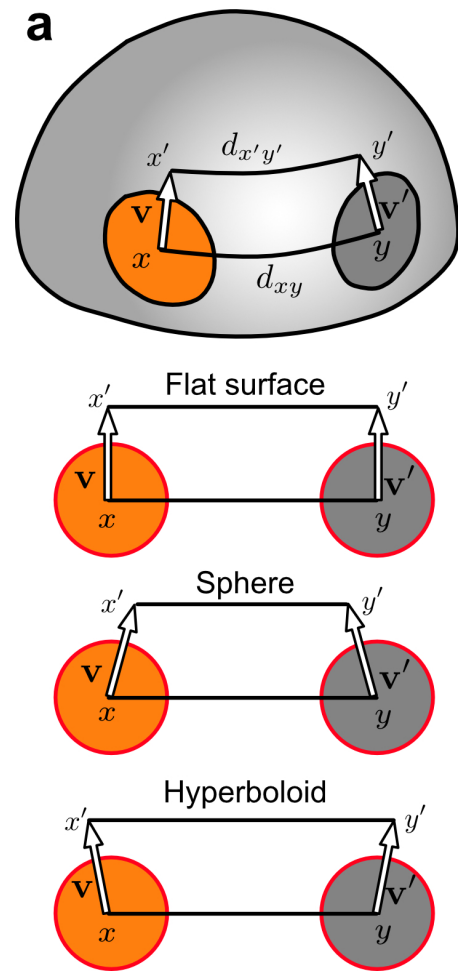

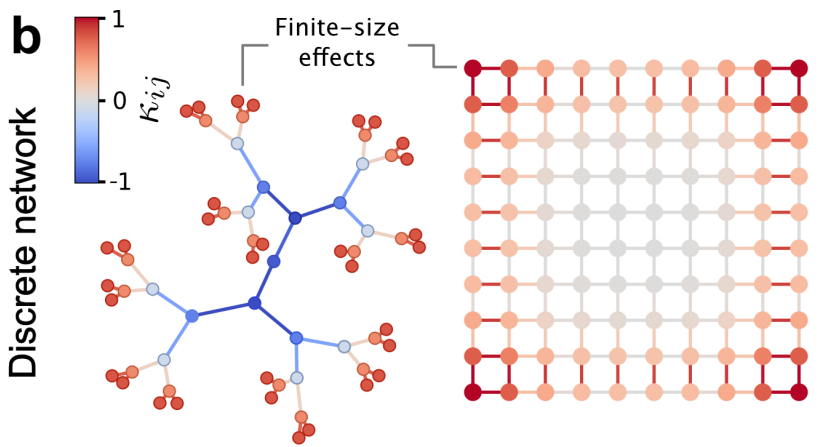

"Tree-like" network

C Negatively curved

Flat
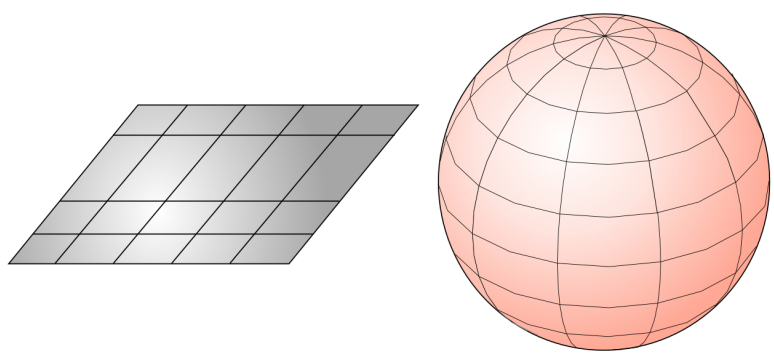

Supplementary Figure 1: Ricci and dynamical Ollivier Ricci curvature on canonical surfaces and graph structures. a Ricci curvature on a manifold. The geodesic distance of close points $x$ and $y$ on average changes when translated by parallel vectors $\mathbf{v}$ and $\mathbf{v}^{\prime}$ on the unit circle in the tangent planes at $x$ and $y$. On planes the points remain equidistant, on spheres the points contract and on hyperbolic surface they expand. $\mathbf{b}$ Canonical graphs with edges coloured by the dynamical OR curvature (Eq. (2)) for $\tau=1$ show that positively and negatively curved graphs have qualitatively different topologies. Away from the boundaries, tree-like topologies are negatively curved, grid-like topologies are flat (zero curvature), whereas clique-like topologies attain positive curvature. Nodes are coloured by the average edge curvature across the neighbours. c Analogously, the differential geometric notion of Ricci curvature is negative on hyperbolic surfaces, zero on planes and positive on spherical surfaces. 


\section{Supplementary Note 1}

In this section, we compute the spectrum of the expected normalised Laplacian matrix of the symmetric $\operatorname{SBM} \mathcal{G}\left(n, k_{\text {in }} / n, k_{\text {out }} / n\right)$. Here $k_{\text {in }}$ and $k_{\text {out }}$ are constants representing the expected number of edges within and across clusters, respectively. The expected adjacency matrix of the symmetric stochastic block model is:

$$
\langle\mathbf{A}\rangle_{\mathcal{G}}=\left(\begin{array}{cc}
\frac{k_{\text {in }}}{n} \mathbf{1}_{n / 2 \times n / 2} & \frac{k_{\text {out }}}{n} \mathbf{1}_{n / 2 \times n / 2} \\
\frac{k_{\text {out }}}{n} \mathbf{1}_{n / 2 \times n / 2} & \frac{k_{\text {in }}}{n} \mathbf{1}_{n / 2 \times n / 2}
\end{array}\right) .
$$

Then, the expected normalised Laplacian matrix is given by

$$
\langle\mathbf{L}\rangle_{\mathcal{G}}=\mathbf{I}-\left(\begin{array}{cc}
\frac{2 k_{\text {in }}}{n\left(k_{\text {in }}+k_{\text {out }}\right)} \mathbf{1}_{n / 2 \times n / 2} & \frac{2 k_{\text {out }}}{n\left(k_{\text {in }}+k_{\text {out }}\right.} \mathbf{1}_{n / 2 \times n / 2} \\
\frac{2 k_{\text {out }}}{n\left(k_{\text {in }}+k_{\text {out }}\right)} \mathbf{1}_{n / 2 \times n / 2} & \frac{2 k_{\text {in }}}{n\left(k_{\text {in }}+k_{\text {out }}\right)} \mathbf{1}_{n / 2 \times n / 2}
\end{array}\right)
$$

The first eigenvector is $\phi_{1}=\mathbf{1}_{n} / \sqrt{n} \in \mathbb{R}^{n}$, with the corresponding eigenvalue being $\lambda_{1}=0$. The second eigenvector has two values, one on each cluster of the SBM. Taking $\phi_{c}(u)=1 / \sqrt{n}$ for $1 \leq u \leq n / 2$ and $-1 / \sqrt{n}$ for $n / 2<u \leq n$ one has asymptotically

$$
\langle\mathbf{L}\rangle_{\mathcal{G}} \phi_{c}=\left[1-\frac{k_{\text {in }}}{k_{\text {in }}+k_{\text {out }}} \frac{2}{n}-\frac{k_{\text {in }}}{k_{\text {in }}+k_{\text {out }}} \frac{2}{n}\left(\frac{n}{2}-1\right)+\frac{k_{\text {out }}}{k_{\text {in }}+k_{\text {out }}} \frac{2}{n} \frac{n}{2}\right] \phi_{c} \stackrel{n \rightarrow \infty}{\longrightarrow} \frac{2 k_{\text {out }}}{k_{\text {in }}+k_{\text {out }}} \phi_{c} .
$$


Figures
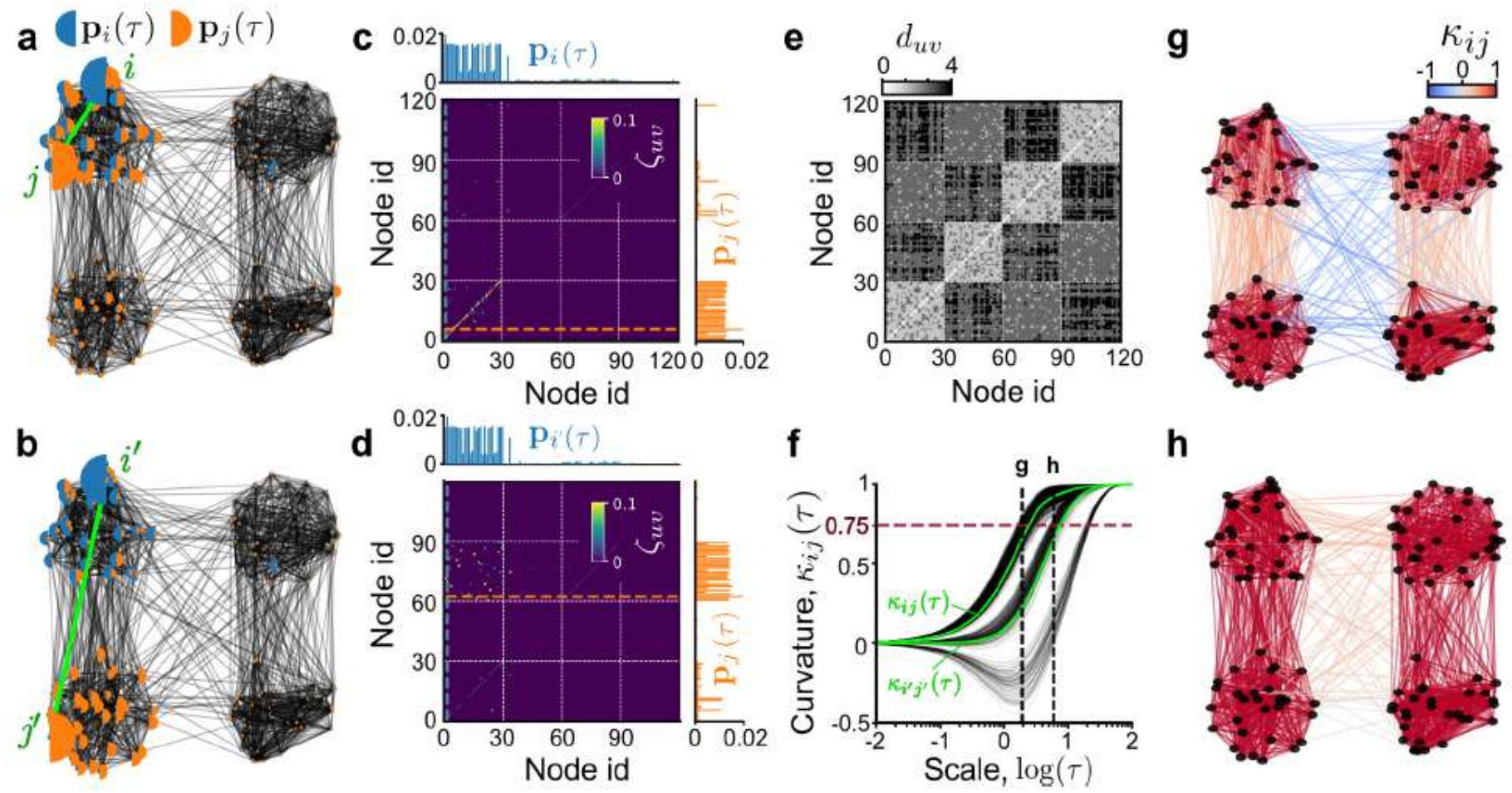

h

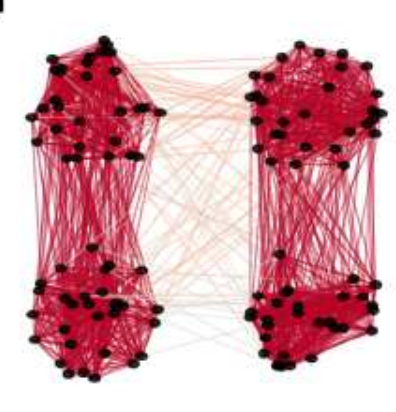

Figure 1

Please see the manuscript file to view figure caption. 
a

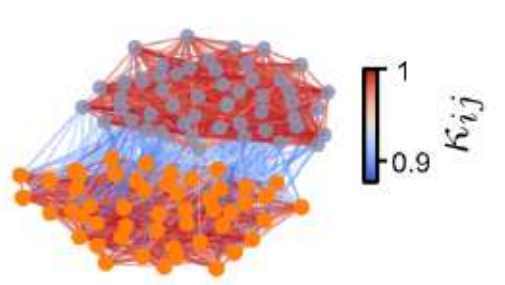

c

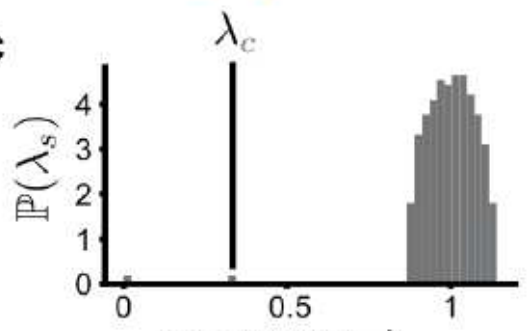

Eigenvalues, $\lambda_{s}$

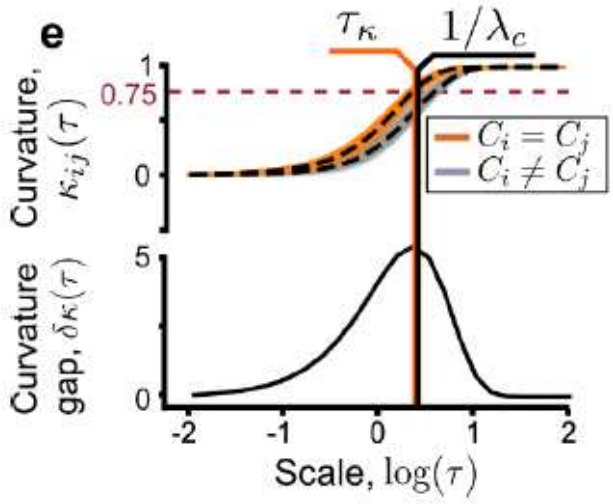

b

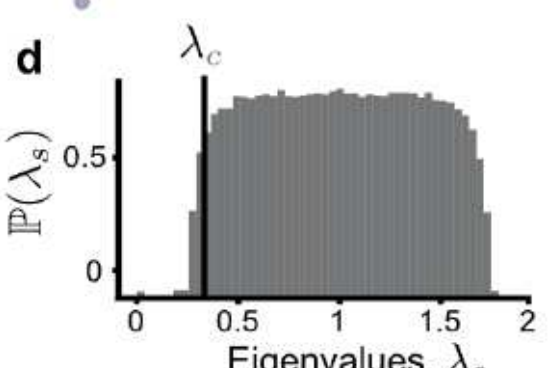

Eigenvalues, $\lambda_{s}$

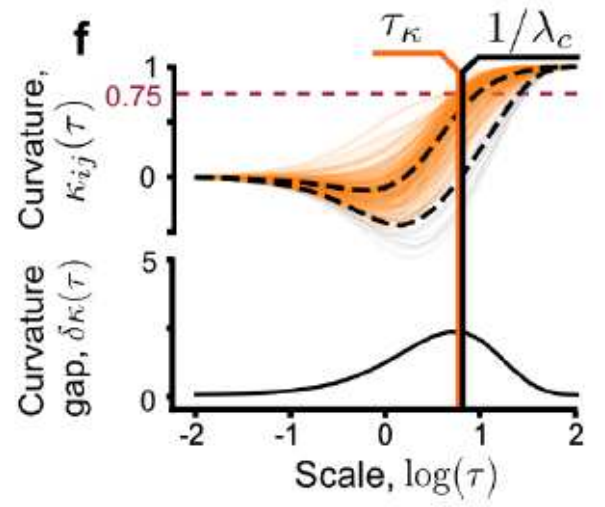

g
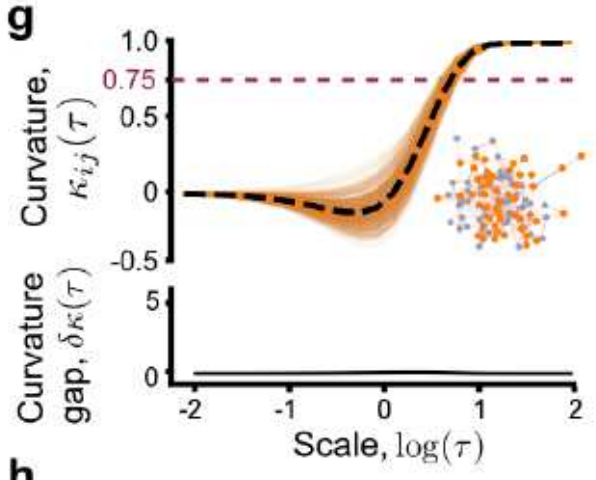

h

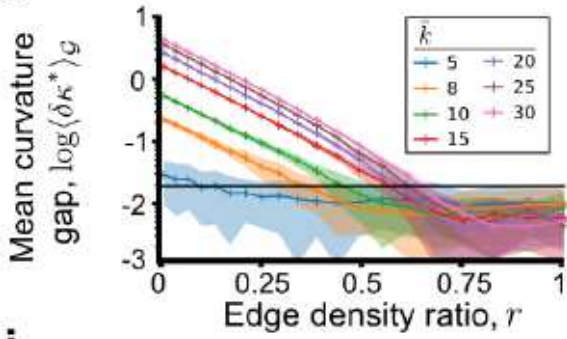

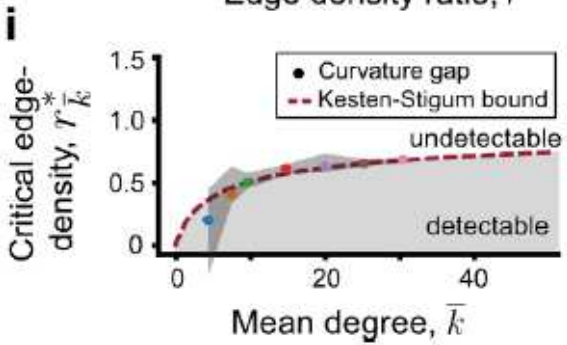

Figure 2

Please see the manuscript file to view figure caption.
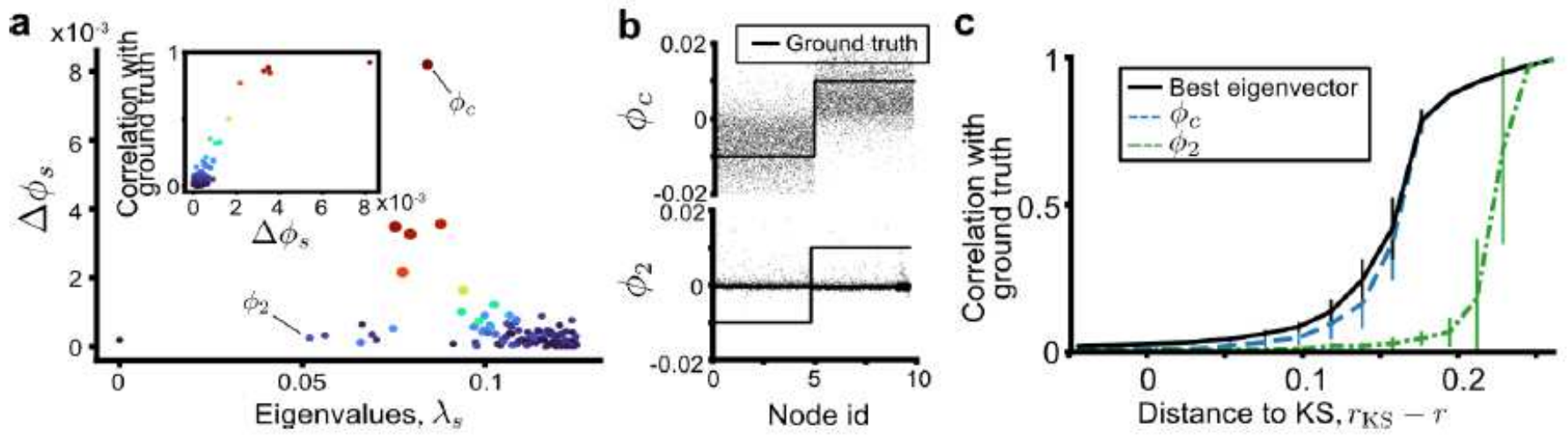

Figure 3

Please see the manuscript file to view figure caption. 

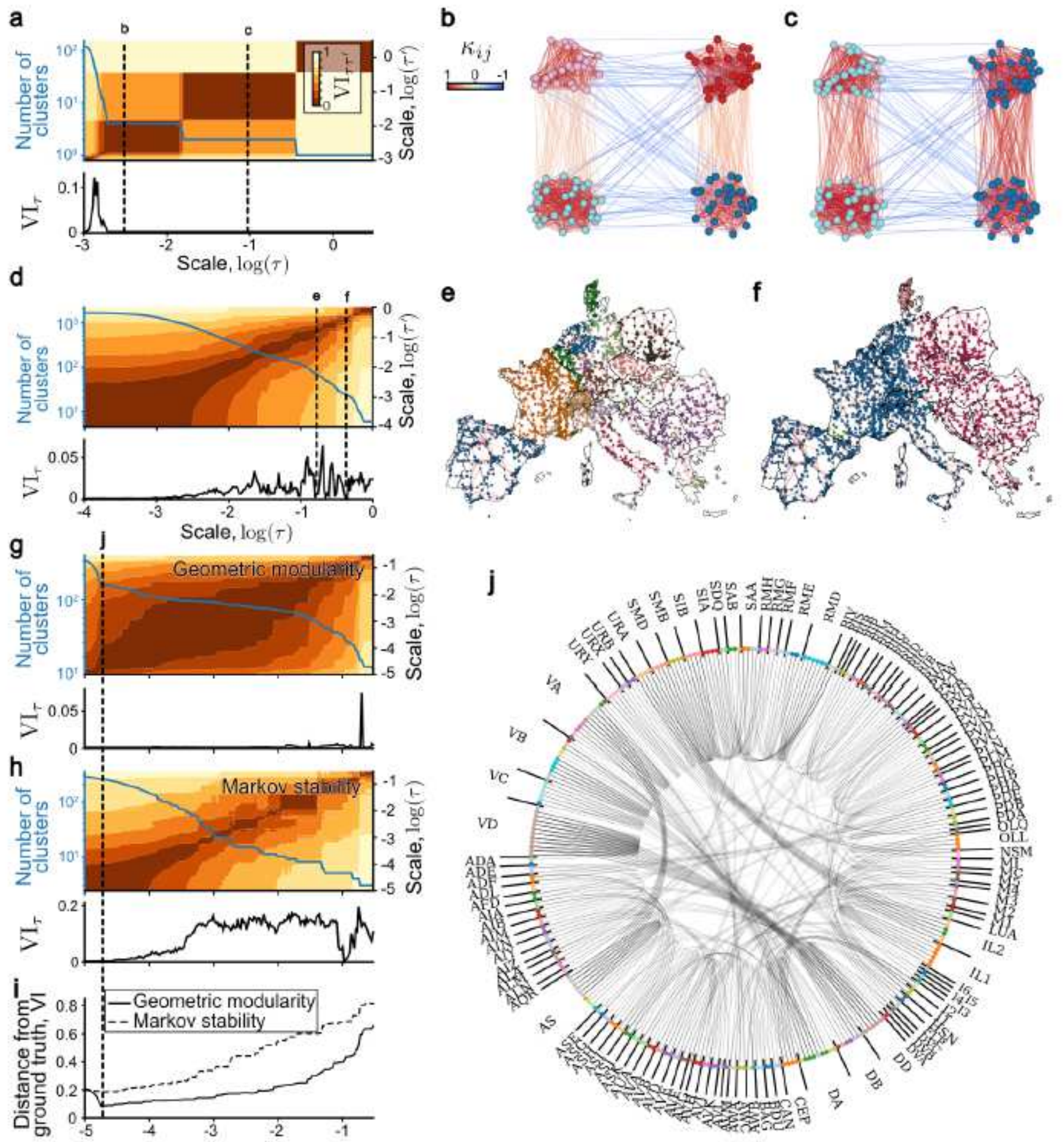

\section{Figure 4}

Please see the manuscript file to view figure caption. 